\title{
Orbital evolution of the Gefion and Adeona asteroid families: close encounters with massive asteroids and the Yarkovsky effect
}

\author{
V. Carruba, ${ }^{\mathrm{a}, *}$ J.A. Burns,${ }^{\mathrm{a}, 1}$ W. Bottke, ${ }^{\mathrm{b}}$ and D. Nesvorný ${ }^{\mathrm{b}}$ \\ ${ }^{a}$ Department of Astronomy, Cornell University, Ithaca, NY 14853, USA \\ ${ }^{\mathrm{b}}$ Department of Space Studies, Southwest Research Institute, Boulder, CO 80302, USA
}

Received 1 May 2002; revised 29 October 2002

\begin{abstract}
Asteroid families are groupings of minor planets identified by clustering in their proper orbital elements; these objects have spectral signatures consistent with an origin in the break-up of a common parent body. From the current values of proper semimajor axes $a$ of family members one might hope to estimate the ejection velocities with which the fragments left the putative break-up event (assuming that the pieces were ejected isotropically). However, the ejection velocities so inferred are consistently higher than $N$-body and hydro-code simulations, as well as laboratory experiments, suggest. To explain this discrepancy between today's orbital distribution of asteroid family members and their supposed launch velocities, we study whether asteroid family members might have been ejected from the collision at low speeds and then slowly drifted to their current positions, via one or more dynamical processes. Studies show that the proper $a$ of asteroid family members can be altered by two mechanisms: (i) close encounters with massive asteroids, and (ii) the Yarkovsky non-gravitational effect. Because the Yarkovsky effect for kilometer-sized bodies decreases with asteroid diameter $D$, it is unlikely to have appreciably moved large asteroids (say those with $D>15 \mathrm{~km}$ ) over the typical family age (1-2 Gyr).

For this reason, we numerically studied the mobility of family members produced by close encounters with main-belt, non-family asteroids that were thought massive enough to significantly change their orbits over long timescales. Our goal was to learn the degree to which perturbations might modify the proper $a$ values of all family members, including those too large to be influenced by the Yarkovsky effect. Our initial simulations demonstrated immediately that very few asteroids were massive enough to significantly alter relative orbits among family members. Thus, to maximize gravitational perturbations in our 500-Myr integrations, we investigated the effect of close encounters on two families, Gefion and Adeona, that have high encounter probabilities with 1 Ceres, by far the largest asteroid in the main belt. Our results show that members of these families spreads in $a$ of less than 5\% since their formation. Thus gravitational interactions cannot account for the large inferred escape velocities.

The effect of close encounters with massive asteroids is, however, not entirely negligible. For about $10 \%$ of the simulated bodies, close encounters increased the "inferred" ejection velocities from sub-100 m/s to values greater than $100 \mathrm{~m} / \mathrm{s}$, beyond what hydro-code and $N$-body simulations suggest are the maximum possible initial ejection velocity for members of Adeona and Gefion with $D>15 \mathrm{~km}$. Thus this mechanism of mobility may be responsible for the unusually high inferred ejection speeds of a few of the largest members of these two families.

To understand the orbital evolution of the entire family, including smaller members, we also performed simulations to account for the drift of smaller asteroids caused by the Yarkovsky effect. Our two sets of simulations suggest that the two families we investigated are relatively young compared to larger families like Koronis and Themis, which have estimated ages of about 2 Byr. The Adeona and Gefion families seems to be no more than 600 and 850 Myr old, respectively.
\end{abstract}

(C) 2003 Elsevier Science (USA). All rights reserved.

Keywords: Asteroids, Asteroid, Dynamics, Orbits, Resonances

* Corresponding author. Fax: +1-607-255-5875.

E-mail address: valerio@astro.cornell.edu (V. Carruba).

${ }^{1}$ Also at Department of Theoretical and Applied Mechanics, Cornell University. 


\section{Introduction}

Nearly a century ago, K. Hirayama noticed that asteroids sometimes cluster at similar locations in proper element space (proper semimajor axis $a$, proper eccentricity $e$, and proper inclination $i$ ). Unlike instantaneous orbital elements, which respond to short-period perturbations, proper elements are nearly constant over long time spans (Lemaitre et al., 1993). They are thus useful to identify asteroids having a common origin. Indeed, the similarity of spectral signatures among members of each group (also called an "asteroid family") suggests a common progenitor that has been collisionally disrupted.

The velocity distribution of ejecta fragments leaving a catastrophic break-up has considerable interest throughout Solar System physics. Ejection velocities can be inferred by (i) measuring the distance between the $(a, e, i)$ center of the family and the $(a, e, i)$ of each family member's orbit, (ii) assuming values of the true anomaly and argument of perihelion of the parent body at the instant of impact and then applying Gauss' equations (Morbidelli et al., 1995) to these values to obtain the velocities at infinity, (iii) computing the escape velocity of each fragment (Cellino et al., 1999), and (iv) quadratically summing velocities at infinity and escape velocities.

The velocities inferred in this way for many known families are $1 \mathrm{~km} / \mathrm{s}$ and more (Cellino et al., 1999). Such speeds are inconsistent with hydro-code and $\mathrm{N}$-body simulations (Michel et al., 2001) that find maximum ejection velocities $v_{\mathrm{ej}}$ of less than $100 \mathrm{~m} / \mathrm{s}$ for $\mathrm{S}$ family members greater than $15 \mathrm{~km}$ in diameter $(D)$ when the ratio between the mass of the largest remnant $\left(M_{\mathrm{LR}}\right)$ and that of the parent body $\left(M_{\mathrm{PB}}\right)$ is 0.04 . This latter value applies to the Koronis family, and is similar to the Gefion family's ratio (0.06). The maximum value of $v_{\mathrm{ej}}$ (as inferred by the method described above) for the same size range in the real Koronis family is $200 \mathrm{~m} / \mathrm{s}$, less than the typical family's value but considerably more than expected theoretically. The same simulations produce values of $v_{\mathrm{ej}}$ of less than $150 \mathrm{~m} / \mathrm{s}$ for those family members with $5<D<15 \mathrm{~km}$, while the corresponding maximum value for this size range in the real Koronis family is $400 \mathrm{~m} / \mathrm{s}$. Ejection velocities are expected to be smaller for higher values of $M_{\mathrm{LR}} / M_{\mathrm{PB}}$, as for Adeona (0.51, Cellino et al., 1999). In those cases where high velocities are obtained from hydro-code models, the fragment distribution is dominated by bodies that would be too small to be observable in asteroid surveys. Low ejection speeds are also seen in laboratory experiments (Fujiwara et al., 1989; Martelli et al., 1994).

To attempt to explain the inconsistency between high inferred launch speeds estimated from observed family members and much lower modeled speeds, we test the hypothesis that family members were launched with relatively low ejection velocities (as the models predict), and then the orbits of family members dynamically evolved to their current locations over long timescales. In this way higher ejection speeds would be inferred from today's orbits (subsequently we will therefore distinguish between "initial" and "inferred" ejection velocities).

Three mechanisms can change one or more of the proper elements of a main-belt asteroid:

(i) Resonances. Several groups (Gladman et al., 1997; Murray and Holman 1997; Migliorini et al., 1998, Morbidelli and Nesvorný, 1999; Nesvorný et al., 2002) have shown that mean-motion, three-body (Murray and Holman, 1997; Murray et al., 1998; Nesvorný and Morbidelli, 1998, 1999), and secular resonances are capable of modifying proper the $e$ and $i$ among main-belt asteroids. The proper $a$ is left unchanged by resonances (Murray and Dermott, 1999).

(ii) The Yarkovsky effect. The Yarkovsky effect happens when solar radiation is absorbed at the body's surface and re-emitted in the infrared band (Burns et al., 1979; Rubincam, 1995; Farinella et al., 1998; Spitale and Greenberg, 2001; Bottke et al., 2002). The thermal radiation from the surface's hottest parts carries away more linear momentum than that from the coldest parts, such that the overall imbalance of the re-emission produces a recoil. This effect will modify the proper $a$ by an amount that depends on various physical and dynamical parameters, including thermal constants, rotation speed, obliquity, orbital geometry, and effective radius. The Yarkovsky effect is size-dependent, and dominates the $a$ dispersion of smaller asteroids (Farinella and Vokrouhlický, 1999).

(iii) Gravitational scattering by large asteroids. Nearly all bodies in the asteroid belt cross the orbits of 1 Ceres, 2 Pallas, 4 Vesta, or 10 Hygiea, and some approach them closely. When mutual encounter velocities are low, the trajectory of a small body making a close fly-by of these much larger asteroids can be gravitationally deflected, with a consequent change in its heliocentric orbital elements. While the probabilities and typical mutual velocities of such encounters are well understood (Farinella and Davis, 1992; Bottke and Greenberg, 1993), only a few recent studies have been carried out on their long-term effects (Carruba et al., 2000, 2001, 2002; Nesvorný et al., 2002).

Several groups have recently incorporated the Yarkovsky effect when considering the dynamics of a few prominent asteroid families. Bottke et al. (2001) integrated the Koronis family, Nesvorný et al. (2002) studied the Flora family, while Vokrouhlický et al. (2002, in preparation) followed the Eos, Eunomia, and Dora families. They all find that the Yarkovsky effect can modify the semimajor axis distribution of the smaller asteroids over timescales comparable to the family ages $(\simeq 1 \mathrm{Gyr})$. However, at multikilometer sizes the Yarkovsky effect varies inversely with the body's diameter and cannot appreciably move large asteroids over such periods of time.

To clarify the meaning of "large" and "small" in this contest, we note that the maximum drift rate (which occurs 
for $0^{\circ}$ and $180^{\circ}$ obliquities) due to the diurnal Yarkovsky effect for a $15-\mathrm{km}$ basaltic object, covered with regolith, is $\simeq 5 \times 10^{-4} \mathrm{AU} / 100 \mathrm{Myr}$ (Farinella and Vokrouhlický, 1999; Vokrouhlický, 1999; Bottke et al., 2001). This Yarkovsky drift rate equals the mean diffusion rate produced by close encounters with the three most massive asteroids, as found by Nesvorný et al. (2002). Thus, we choose $D=15$ $\mathrm{km}$ as the separation between "large" and "small," meaning that once $D \geq 15$ close encounters should be the dominant mechanism of $a$-mobility due. We caution, however, that the precise size at which close encounters prevails over the to the Yarkovsky effect is poorly constrained because the actual physical parameters characterizing the Yarkovsky force are not well determined.

The question that therefore remains to be addressed concerns the semimajor axis distribution of the largest bodies: are the values of $a$ for these bodies primordial or have they been modified after the family's formation? Since capture into resonances does not modify the proper $a$, we may use the distribution of proper semimajor axes to infer the assumed-isotropic ejection velocity fields of the largest members of asteroid families (Cellino et al., 1999). This approach will be correct only if we can demonstrate that massive asteroids are unable to significantly alter the orbits of most of the largest members of asteroid families.

Therefore this paper will investigate the extent to which the proper elements, and in particular the semimajor axes, of asteroid family members in the central main belt can be affected by the above-mentioned effects. We first focus our study on the mobility due to close encounters with massive asteroids because this could be the only mechanism able to significantly modify the proper $a$ of large bodies over family lifetimes. At the paper's end we consider how the Yarkovsky effect moves smaller family members in order to provide another constraint on the family ages.

\section{Methods}

To account for mutual asteroidal perturbations in the main belt, we first had to decide what objects are important (in particular, is most of the semimajor axis mobility due to perturbations from the four largest asteroids, or should we also consider the cumulative effect of all 682 asteroids larger than $50 \mathrm{~km}$ ?). To address this question, we tracked the evolution of 30 test particles in the intermediate belt ( $a$ $=2.6-2.8 \mathrm{AU}, e=0.1, i=0^{\circ}-15^{\circ}$ ) for $100 \mathrm{Myr}$ under the influence of(i) all 682 asteroids of radius larger than $50 \mathrm{~km}$, (ii) the four largest asteroids 1 Ceres, 2 Pallas, 4 Vesta, and 10 Hygiea, and (iii) all asteroids larger than $50 \mathrm{~km}$, except the four largest ones. Rather than employ direct numerical integrations for these tests, which would be computationally expensive, we applied a Monte Carlo code based on the Öpik-Arnold method (Öpik, 1951), as implemented by Melosh and Tonks (1993) (see also Dones et al., 1999).

We used a value of $4.70 \times 10^{-10} M_{\text {Sun }}$ for the mass of
1 Ceres, of $1.21 \times 10^{-10} M_{\text {Sun }}$ for 2 Pallas, and of $1.36 \times$ $10^{-10} M_{\text {Sun }}$ for 4 Vesta (Michalak 2000; the errors on the determination of the masses were $\pm 0.04,0.26$, and $0.05 \times$ $10^{-10} M_{\text {Sun }}$, respectively). The value for the mass of 10 Hygiea, $0.47 \times 10^{-10} M_{\text {Sun }}\left( \pm 0.23 \times 10^{-10} M_{\text {Sun }}\right)$, was determined by Scholl et al. (1987). We estimated the masses of the remaining 678 asteroids larger than $50 \mathrm{~km}$ by first determining their diameters through the equation

$$
D(\mathrm{~km})=1329 \frac{10^{(-H / 5)}}{\sqrt{A}},
$$

where $H$ and $A$ are the absolute magnitude and albedo (Bowell et al., 1989; a value of the albedo typical of the spectral type ( 0.05 for C-type and 0.10 for S-type) was used for asteroids lacking this information). The masses were determined assuming that the asteroids were spheres of uniform density and using values of the mean density typical of their spectral type $\left(2500 \mathrm{~kg} / \mathrm{m}^{3}\right.$ for S-type bodies, $1300 \mathrm{~kg} / \mathrm{m}^{3}$ for C-type).

Fig. 1 shows the changes in semimajor axis of the 30 test particles in the three cases. While the four largest asteroids alone moved 25 particles (corresponding to $83 \%$ of the integrated objects) by more than $10^{-4} \mathrm{AU}$, with a maximum displacement of $1.2 \times 10^{-3} \mathrm{AU}$, the remaining 678 asteroids could push only 2 bodies ( $7 \%$ of our sample) by more than $10^{-4} \mathrm{AU}$ over the length of the integration. Thus, perturbations from other than the four largest asteroids should be negligible.

To understand the influence of the largest asteroids in the main-belt population, we also turn to Nesvorný et al. (2002), who integrated 300 asteroids in the main belt over $120 \mathrm{Myr}$ using the Mercury integrator (Chambers, 1999), under the influence of 7 planets and the three largest asteroids (1 Ceres, 2 Pallas, 4 Vesta). They found that most of the asteroidal semimajor-axis evolution was due to close encounters with 1 Ceres. The standard deviation of changes in $a$, defined as

$$
\sigma_{a}(t)=\sqrt{\frac{\sum_{j}\left[a_{j}(t)-a_{j}(0)\right]^{2}}{N-1}}
$$

(with $N$ the number of objects), followed a power law of the form

$$
\sigma_{a}(t)=C t^{B},
$$

with an exponent $B=0.63$. Changes in $a$ grow faster than a random walk law $(B=0.5)$ since there are encounters with repeated geometries, asymmetries in the perturbations, etc. For $a>2.5$ AU Nesvorný et al. (2002) found an enhanced effect of encounters with 1 Ceres and a larger value of the exponent $(B=0.68)$.

According to these results, the long-term evolution of asteroid families due to close encounters depends on their positions in the asteroid belt. In particular, asteroid families near 1 Ceres, which Nesvorný et al. (2002) and our study identify as the main perturber, should have higher frequen- 
A

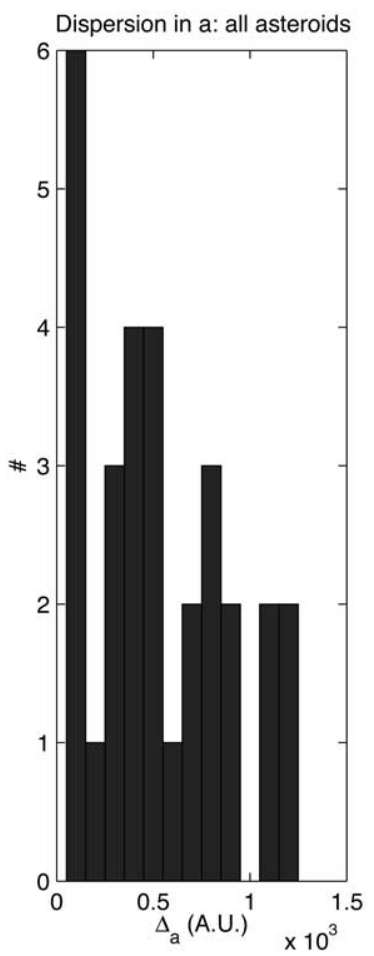

B

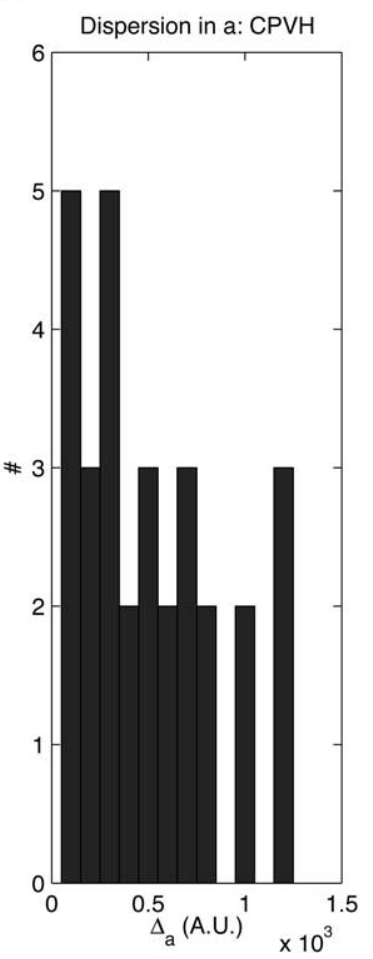

C

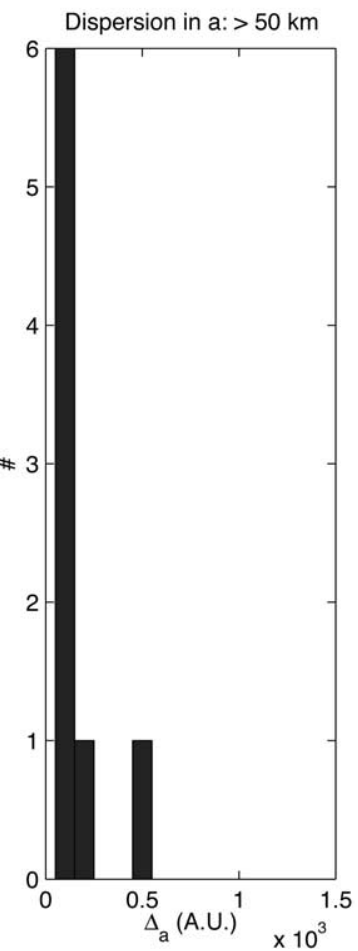

Fig. 1. Histograms of changes in the semimajor axis of 30 test particles integrated over 100 Myr under the influence of (a) all 682 asteroids of radius larger than $50 \mathrm{~km}$, (b) 1 Ceres, 2 Pallas, 4 Vesta, and 10 Hygiea (CPVH in the figure), and (c) the remaining 678 bodies.

cies of encounters at lower mutual velocities, resulting in the most significant changes in $a$. To test this hypothesis in an approximate manner, we used a code based on the approach of Greenberg (1982), who assumes that the gravitational interaction between two closely approaching orbiting bodies can be modeled as a two-body hyperbolic encounter. We used this code to compute $\left(\left\langle\Delta a^{2}\right\rangle / T\right)^{1 / 2}$ (where $\Delta a$ is the change in $a$ and $T$ is the orbital period of the perturbee) for 1 Myr for an $(a, e)$ grids of test bodies surrounding 1 Ceres, 2 Pallas, 4 Vesta, and 10 Hygiea. In particular, we have chosen values for the test particles in the range of 2 to $4 \mathrm{AU}$ for the cases of 1 Ceres, 2 Pallas, and 10 Hygiea, and between 1.5 and 3.9 AU for 4 Vesta. The test eccentricities ranged between 0 and 0.3 for 1 Ceres, 4 Vesta, and 10 Hygiea and between 0 and 0.45 for 2 Pallas, to take into account its larger eccentricity $\left(e_{\text {Pallas }}=0.277\right)$.

For low inclinations, we found that 1 Ceres is by far the most effective perturber across the intermediate belt $(a=$ 2.6-3.0 AU, $e=0.0-0.3$ ), but 2 Pallas has a comparable effect to 1 Ceres for $i>20^{\circ}$. Fig. 2 shows contour plots of $\left(\left\langle\Delta a^{2}\right\rangle / T\right)^{1 / 2}$ for $i_{0}=15^{\circ}$ for 1 Ceres (similar plots for the other three largest asteroids are not shown to save space). In Fig. 2 we also display the locations of the centers of the Adeona and Gefion families because these families lie closest to 1 Ceres and hence have experienced drift rates of more than $\left(\left\langle\Delta a^{2}\right\rangle / T\right)^{1 / 2} \simeq 6 \times 10^{-6} \mathrm{AU} / \mathrm{Myr}^{0.5}$, among the largest rates in the middle main belt. Based on these results, we choose these two families to investigate the long-term effect of gravitational scattering from massive asteroids.

The Monte Carlo code we used can provide insights into the long-term effects of close encounters with massive asteroids. However, this kind of approach is, by definition, approximate, since some important effects (e.g., the alignment of the apsides and nodes between perturber and test body, mean-motion, and secular resonances) cannot be treated easily (Dones et al., 1999; Carruba et al., 2000). For this reason, we turn to the full $N$-body, symplectic algorithm known as SyMBA (Duncan et al., 1998; Levison and Duncan, 2000). SyMBA has the speed of highly efficient computer algorithms using Wisdom-Holman mapping (Wisdom and Holman, 1991), but it can also accurately handle close encounters by employing a variant of established multiple step-size techniques (Biesiadecki and Skeel, 1993). When two bodies suffer a mutual encounter, the step-size for the relevant bodies is recursively subdivided to whatever level is required to maintain accuracy. This method is fast and accurate enough to allow us to integrate close encounters between large asteroids and test bodies while also including the perturbations of the major planets.

For the integrations with SyMBA we choose to deal with two kinds of simulated families. In one case we used actual members of the Adeona and Gefion families; in the other we generated a distribution of test particles that simulated the initial conditions immediately after the parent body's break- 


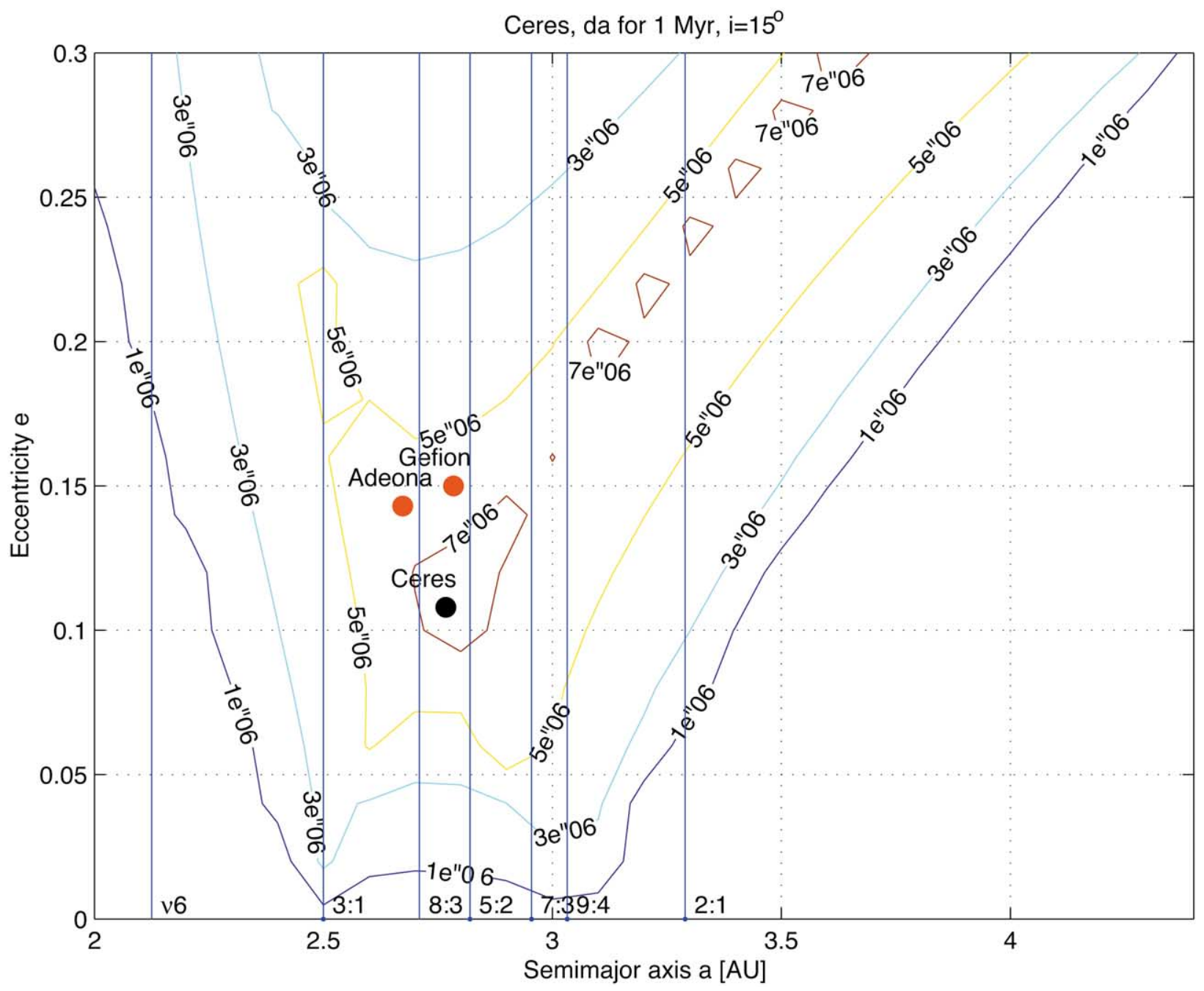

Fig. 2. Contour plots of $\left(\left\langle\Delta a^{2}\right\rangle / T\right)^{1 / 2}$ (=da in the figure, $T$ is the orbital period of the perturbee) for test particles perturbed by 1 Ceres. As identified along the bottom axis, the vertical lines show the locations of the strongest mean-motion resonances with Jupiter, and the approximate position of the $\nu_{6}$ secular resonance. The dots indicate Ceres' location plus the positions of the centers of the Adeona and Gefion families.

up. We called these two sets of test particles "real" and "synthetic" families. We wanted to use real members because by determining the time dependence of the dispersion in $e$ and $i$ due to chaotic diffusion in mean motion and three-body resonances of real members, it is possible (Nesvorný et al., 2002) to set limits on the age of the family (see Section 5). On the other hand, we wished to investigate how the orbital elements of family members evolved with time after the parent body's break-up, and this can be done only by simulating the initial conditions following the family formation.

To set up our integrations with real members, we employed members of the families as determined by Zappalà et al. (1995), who applied a hierarchical clustering method (HCM) to a proper-element database containing 12,487 numbered and unnumbered main-belt asteroids known as of 1994. We only considered asteroids with certain membership (Cellino's classes QC $=2$ or 3, Zappalà et al., 1994,
1995). The sizes (effective diameters) were determined by using the absolute magnitude $(H)$ and Bond albedo $(A){ }^{2}$

Though useful, the set of proper elements used by Zappalà et al. (1995) is incomplete, since most of the asteroids in the proper element catalog available at the time had magnitudes $H<12.5$. Families determined from this cata$\log$ do not include most of the objects with diameters smaller than $\simeq 10 \mathrm{~km}$, since most of these objects were discovered after 1994. Nevertheless, we choose to use such families because(i) they were the only ones available when we initiated our integrations, and (ii) we were interested in the long-term effect of close encounters with massive asteroids on large members (i.e., those objects with $D>15$ $\mathrm{km}$ for which the Yarkovsky effect is relatively ineffective), and for these sizes the Zappalà catalog is complete.

\footnotetext{
${ }^{2}$ Where available, otherwise the average value of the family's albedo was employed.
} 
Thus our integrations had 66 and 84 members of the Adeona and Gefion families, respectively. For objects at smaller sizes $(D<10 \mathrm{~km})$, updated sets of 66,089 analytically computed (Milani and Knežević, 1994) and 31,502 numerically computed (Knežević and Milani, 2000) proper elements are now available. These sets contain also proper elements for asteroids discovered after 1994.

These objects will be relevant for our Yarkovsky integrations, since this effect is the predominant mechanism of $a$ mobility at smaller sizes. To find new members of the Adeona and Gefion families among the asteroids discovered after 1994, we used the HCM method and the state-of-the art proper-element database found at the AstDys web site. ${ }^{3}$ The criterion of family membership requires that all family members are connected by a chain, where each member is attached to its neighbor by less than a limiting "velocity" distance (cutoff). The distance $d$ is defined by a metric in proper-element space, while the cutoff, which Zappalà et al. (1994) define as a Quasi-Random Level (QRL), is a typical minimum distance of background objects in the family's region. Please keep in mind that, while $d$ has dimension of meters per second, it has nothing to do with ejection velocities; rather it is just a way to express distances in properelement space. For our families we adopted the distance metric from Zappalá et al. (1994):

$$
d=n a \sqrt{1.25\left(\frac{\Delta a}{a}\right)^{2}+2(\Delta e)^{2}+2(d \sin \Delta i)^{2}} .
$$

This is a metric in Euclidean space, where $\Delta a, \Delta e$, and $\Delta i$, distances between the proper elements of the asteroid and those of the center of the family, are weighted by particular coefficients, and $n$ is the mean motion. The choice of the cutoff was more arbitrary. Since the number of known asteroids has dramatically increased since 1994, the value of QRL used by Zappalà et al. (1994) is now too high (the average distance between asteroids is now much closer). For our cases we choose a value of $d_{\text {cutoff }}=80 \mathrm{~m} / \mathrm{s}$, which empirically seemed to best fit the current distributions of Adeona and Gefion. Larger values $\left(d_{\text {cutoff }}>100 \mathrm{~m} / \mathrm{s}\right)$ significantly increase the number of outliers, and actually include known members of other families into Adeona and Gefion. Smaller values are unable to account for the population of asteroids with $a<2.65 \mathrm{AU}$ (approximate location of the 11/4 mean-motion resonance with Jupiter), already identified by Zappalà et al. (1994) as Adeona members, and for those asteroids with $a<2.754 \mathrm{AU}$ (13/5 mean-motion resonance with Jupiter) for Gefion. We used the families generated by this procedure ("Nesvorný's families," as opposed to "Zappalá's families") to estimate properties like the standard deviation in proper elements, and the population of asteroids at diameters smaller than $10 \mathrm{~km}$.

While integrations of existing objects can provide useful insights, they cannot tell us about how the distribution has

\footnotetext{
${ }^{3}$ http://hamilton.dm.unipi.it/cgi-bin/astdys/astibo
}

evolved since the families break-up. For this reason, we also generated a distribution of test particles that simulates a possible set of initial conditions immediately after the parent body's disruption ("synthetic families"). A description of the procedure that was used is given in the Appendix. The size distribution was obtained by placing the particles in 8 equally spaced bins in $1 / r$, between a minimum radius of 1 $\mathrm{km}$ and a maximum radius corresponding to the tenth largest asteroid in the family. The cumulative size distributions for the two synthetic families can be fitted by an exponent of $-0.42 \pm 0.03$. The steep size distribution function of the fragments we generated is also suggested by recent hydrocode simulations (Michel et al., 2001). We generated 60 particles for the synthetic Adeona family and 75 particles for the synthetic Gefion family.

These synthetic and real members of the Adeona and Gefion families were integrated for $500 \mathrm{Myr}$ under the gravitational perturbations of the four giant planets and the four most massive asteroids. We used a maximum (this value may be reduced during close encounters) time step of 18 days. Our choice of the time step is conservative. An empirical rule of thumb is that the time step should be at least 30 times less than the smaller period among the simulated bodies, which, in our case, is the orbital period of 4 Vesta. Our time step is $\simeq 70$ times smaller than Vesta's period of 3.61 years.

We tested our integrator with this time step by performing several runs over $100 \mathrm{Myr}$ with the Sun, 1 Ceres, and 20 test particles on orbits similar to 1 Ceres, but with slightly different values of the semimajor axis $( \pm 0.01 \mathrm{AU})$, and 10 different values of mean anomalies, equally spaced from $0^{\circ}$ to $360^{\circ}$. These particles experienced repeated close encounters with 1 Ceres during the simulations, but the Jacobi constant did not change by more than 1 part in $10^{7}$.

Results of our simulations with real and synthetic members of the Adeona and Gefion families will be discussed in the following sections.

\section{Changes in semimajor axis due to close encounters with large asteroids}

In this section we investigate the degree to which close encounters disperse the semimajor axis distribution of family's members (i.e., the time behavior of $\sigma_{a}(t)$ ) and determine the mean drift rates for the Adeona and Gefion families.

We set our SyMBA runs to produce osculating elements every 1000 years. In order to have a first approximation of proper elements, we averaged the osculating elements with a running window of $10 \mathrm{Myr}$ and a shift of $10^{5}$ years following the same approach as Morbidelli and Nesvorný (1999). The integration time was $500 \mathrm{Myr}$ for the real and synthetic members of the Adeona family. We call the output of this procedure "averaged" elements.

Fig. 3a and $\mathrm{b}$ display the time evolution of the mean 
A

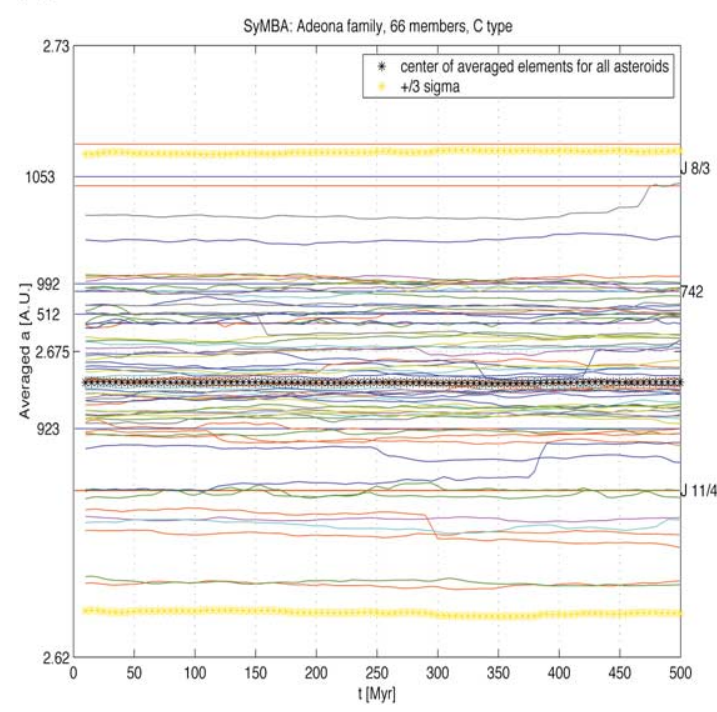

C

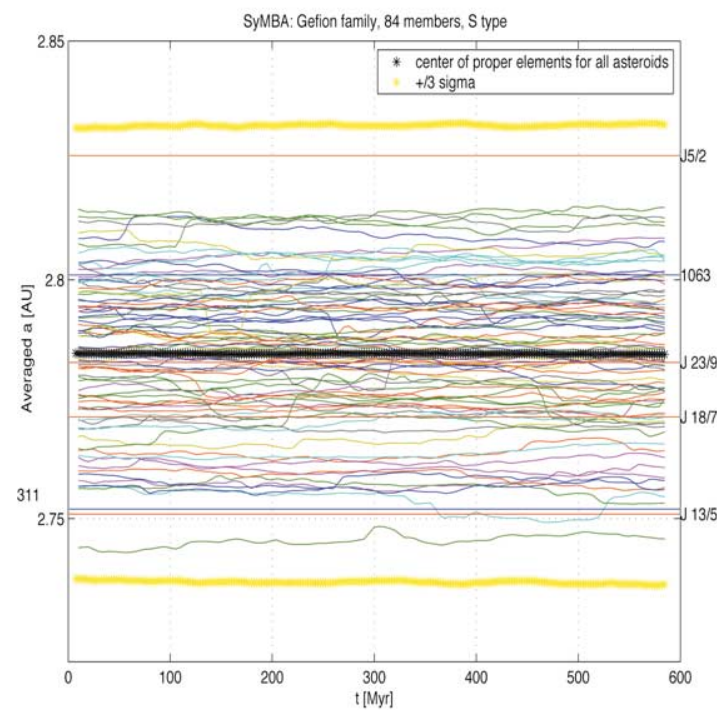

B

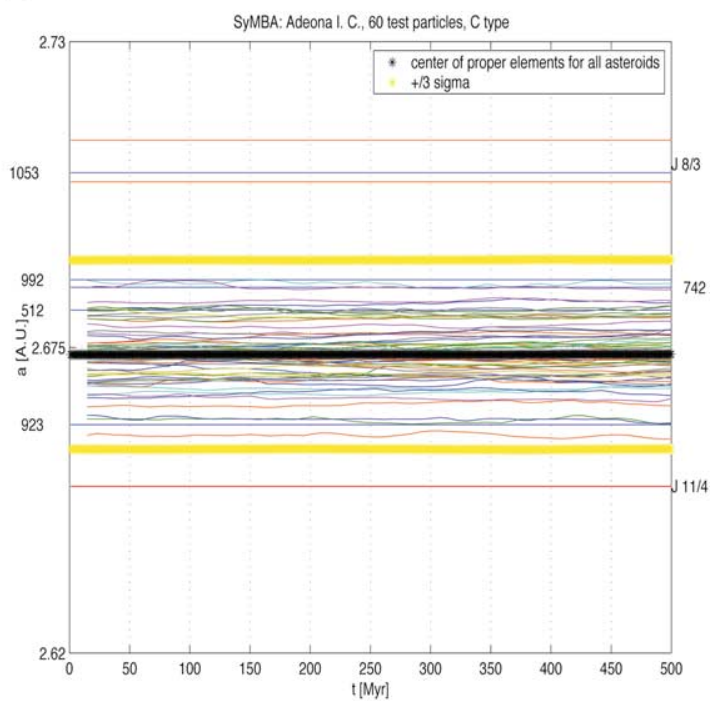

D

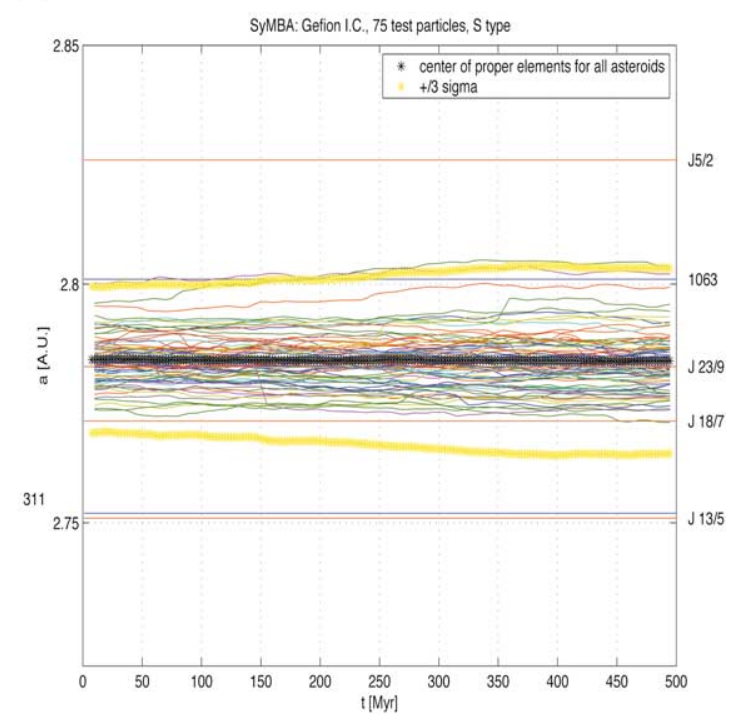

Fig. 3. Time evolution of $a$ for the integrations with real members and synthetic particles for the Adeona (a, b) and Gefion (c, d) families, as perturbed by the giant planets and the four largest asteroids. Horizontal lines show the position of mean-motion resonances with Jupiter and three-body resonances (listed on the edges of each figure). We also plot the center of the family (in black) and the $3 \sigma$ levels from the center. The integration time was of $500 \mathrm{Myr}$.

semimajor axis for our numerical experiment with the Adeona members.

Adeona is bounded by Jupiter's $8 / 3$ mean-motion resonance at $2.705 \mathrm{AU}$, and by the $11 / 4$ at $2.65 \mathrm{AU}$. Close encounters seem to not significantly alter the distribution in the semimajor axis of the family's members. Variations of the standard deviation in $a$ were of 2.9 and $2.7 \%$ at the end of the integration for the real and synthetic family, respectively. Variations of $\sigma_{a}$ may seem larger in Fig. 3a for the real members compared with the corresponding variations for synthetic members (Fig. 3b) because the synthetic family starts a factor of 3 more compact than the real family.

We integrated real and synthetic members of the Gefion family for $500 \mathrm{Myr}$ (Fig. 3c and d). Gefion is crossed by
Jupiter's $18 / 7$ and $23 / 9$ mean-motion resonances, and is close to the $5 / 2$ at $2.82 \mathrm{AU}$. Most of the Gefion members have inclinations near Ceres's $i\left(10.58^{\circ}\right)$ and high collision probabilities (the members of the Gefion family we used in our run had a mean collision probability with 1 Ceres of 6 $\times 10^{-18} \mathrm{~km}^{-2} \mathrm{yr}^{-1}$, with a maximum of $10^{-17} \mathrm{~km}^{-2} \mathrm{yr}^{-1}$, while the average intrinsic collision probability between $D$ $>50 \mathrm{~km}$ asteroids in the main belt is of $2.85 \times 10^{-18}$ $\mathrm{km}^{-2} \mathrm{yr}^{-1}$ (Farinella and Davis, 1992; Bottke et al, 1994)). We find that this family may have been significantly affected by close encounters with 1 Ceres: while the standard deviation in $a$ for the real members changed by 3.3\% at the end of the integration, there was an increase of $30 \%$ for the synthetic family. The fact that those members experienced a 
A

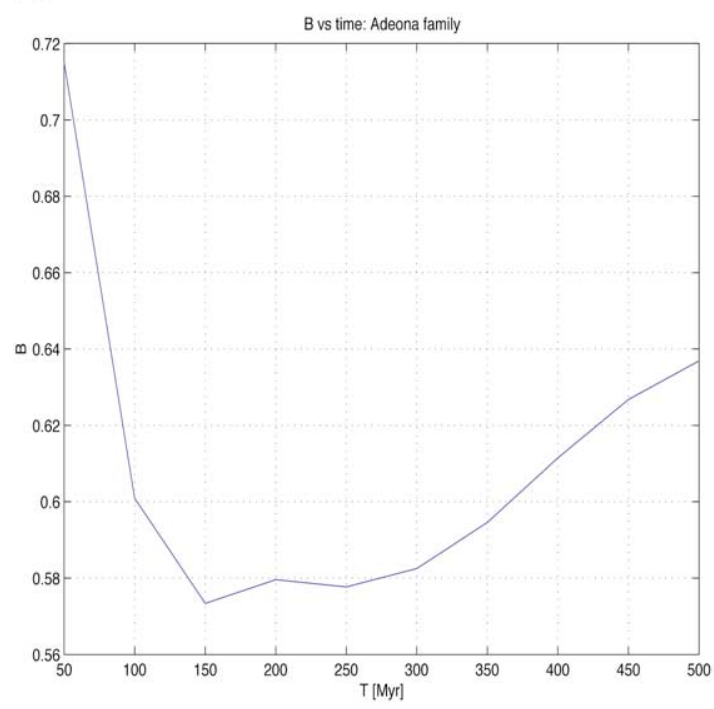

c

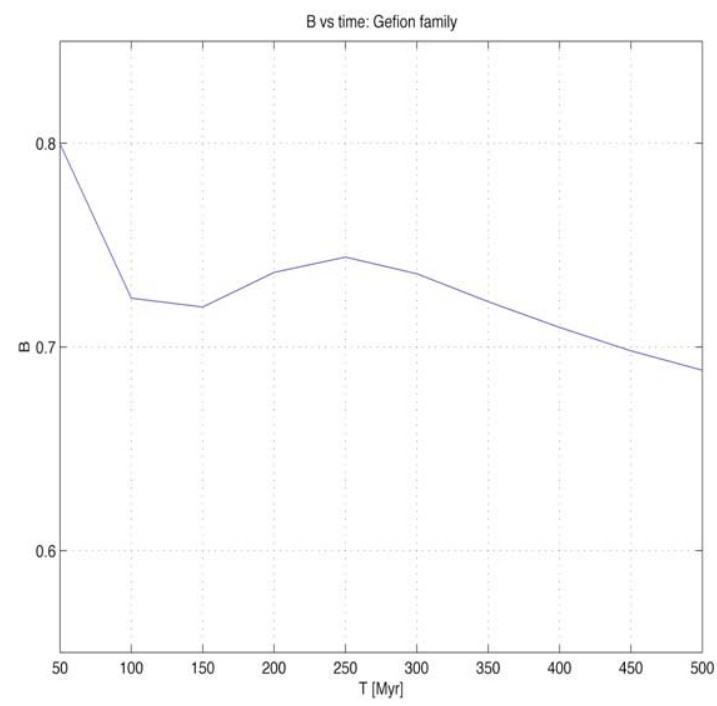

B

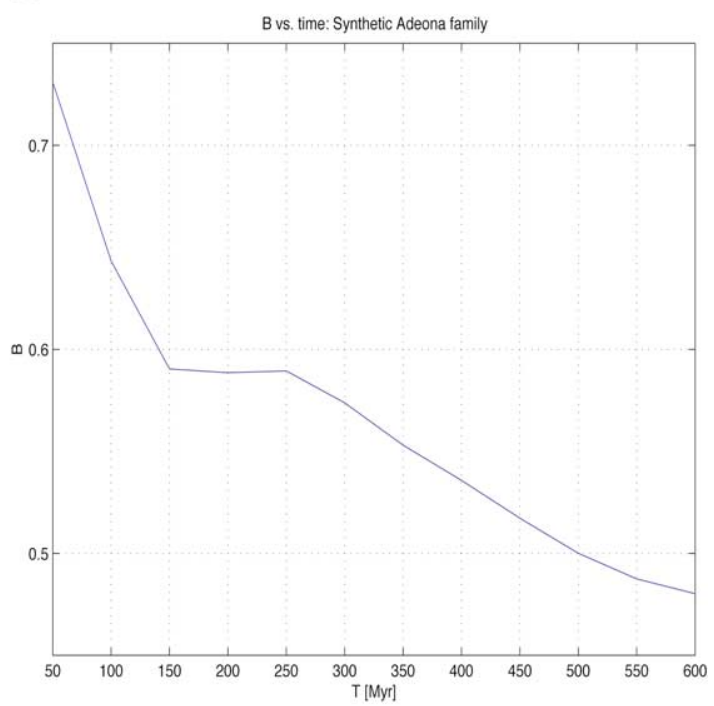

D

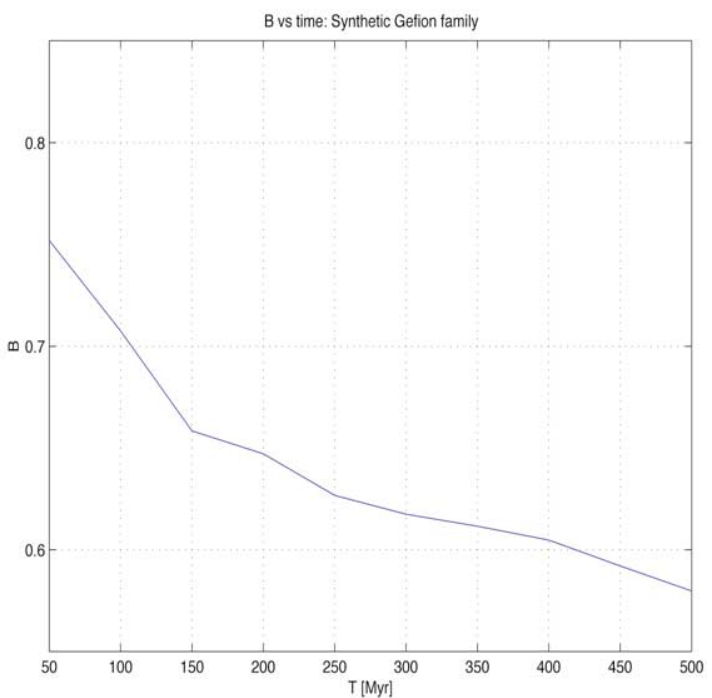

Fig. 4. Time dependence of dispersion rates in $a$ for the real and synthetic members of the Adeona (a, b) and Gefion (c, d) families perturbed by the four giant planets and four largest asteroids. Assuming that the standard deviation of changes in $a$ followed the power law $\sigma_{a}=C t^{B}$, the plots show how $B$ changed with time. $B$ was obtained by performing a least-squares fit every $50 \mathrm{Myr}$, considering all the times that went before.

larger dispersion in $a$ than real members is characteristic of power laws with exponents less than 1 , for which an initial phase of fast expansion is followed by a slower long-term dispersion.

We were also interested in the time dependence of dispersion rates for these asteroid families. Assuming that the standard deviation of changes in $a$ approximately followed Eq. (3), we wanted to find how $B$ changed with time. This is important because in previous works Carruba et al. (2000, 2002) simply extrapolated values of $B$ found by analyzing results of a 120-Myr Mercury integration (300 asteroids in the central main belt under the influence of seven planets and the three largest asteroids) to longer timescales to try to obtain an estimate of the initial dispersion in $a$ of the largest
$(D>20 \mathrm{~km}$ ) members of four asteroid families (Eos, Eunomia, Koronis, and Themis). We wanted to check whether the effect of close encounters can be safely approximated by a power law even for timescales longer than 120 Myr, and determine the uncertainties on $B$ 's values.

Since the dispersion rates vary in different regions of the main belt, it is also important to investigate whether $B$ 's value changes with the asteroid location. Fig. 4 plots $B$ 's time evolution for the simulations involving close encounters. $B$ was computed by a least-squares fit every $50 \mathrm{Myr}$, considering all the times that went before. For the real members of Adeona, $B$ drops from an initial value of 0.71 (errors on the values of $B$ are \pm 0.01 ) to a minimum of 0.57 at $t=150 \mathrm{Myr}$, and then slowly increases to 0.63 . For 
members of the synthetic family, $B$ falls from its initial value of 0.71 to a minimum of 0.48 (less than a random walk) at the end of the simulation. For members of the Gefion family, $B$ oscillates around 0.72 , while for the synthetic family $B$ decreases to a final value of 0.58. Except for the integration with real members of Adeona, $B$ usually decreases with time.

In an integration of 300 main-belt asteroids, Nesvorný et al. (2002) found that the spreading of the families by massive asteroids followed a power law with $B>0.5$, just as we have. They concluded that encounters did not necessarily produce a random walk, because encounters may have repeated geometry or asymmetries in the perturbations. Our work supports this interpretation. Furthermore, we believe that the fact that $B$ in general decays with time may result from repeated close encounters that place the test objects onto orbits that have lower collision probabilities. Since $B$ changes nonlinearly with time, it is unsafe to extrapolate our drifting rate to times longer than our integrations $(\simeq 500$ Myr).

In this work we were mainly interested in investigating the changes in the semimajor axis produced by close encounters with massive asteroids on asteroid family members. Figure 5a and 6a show histograms of changes in $a$ at 100,300 , and $500 \mathrm{Myr}$ for the simulations with synthetic families. We used a bin size of $a=0.0002$ AU. We also computed the ejection velocities of the synthetic members by using Gauss' equations for the velocity at infinity

$$
\begin{aligned}
\delta a / a= & \frac{2}{n a\left(1-e^{2}\right)^{1 / 2}}\left[(1+e \cos f) \delta V_{\mathrm{T}}\right. \\
& \left.+(e \sin f) \delta V_{\mathrm{R}}\right], \\
\delta e= & \frac{\left(1-e^{2}\right)^{1 / 2}}{n a}\left[\frac{e+2 \cos f+e \cos ^{2} f}{1+e \cos f} \delta V_{\mathrm{T}}\right. \\
& \left.+(\sin f) \delta V_{\mathrm{R}}\right], \\
\delta i= & \frac{\left(1-e^{2}\right)^{1 / 2} \cos (\omega+f)}{n a} \delta V_{\mathrm{W}},
\end{aligned}
$$

where $f$ and $\omega$ are the true anomaly and argument of pericenter at the instant of impact and $\left(\delta V_{\mathrm{T}}, \delta V_{\mathrm{R}}, \delta V_{\mathrm{W}}\right)$ are the components of $\delta V$ along the direction of motion, in the radial direction, and perpendicularly to the orbital plane, respectively (Morbidelli et al., 1995). We used the values of mean anomaly and argument of pericenter at the moment of destruction employed to generate the synthetic families, and Cellino et al. (1999)'s approach for the escape velocities, i.e,

$$
V_{\mathrm{esc}}=\sqrt{\frac{8 \pi G \rho}{3}} R_{\mathrm{PB}}\left(1-\frac{D_{i}}{R_{\mathrm{PB}}}\right),
$$

where $\rho$ is the density of the parent body $\left(2500 \mathrm{~kg} / \mathrm{m}^{3}\right.$ for S-type bodies, $1300 \mathrm{~kg} / \mathrm{m}^{3}$ for C-type), $R_{\mathrm{PB}}$ is the radius of
A
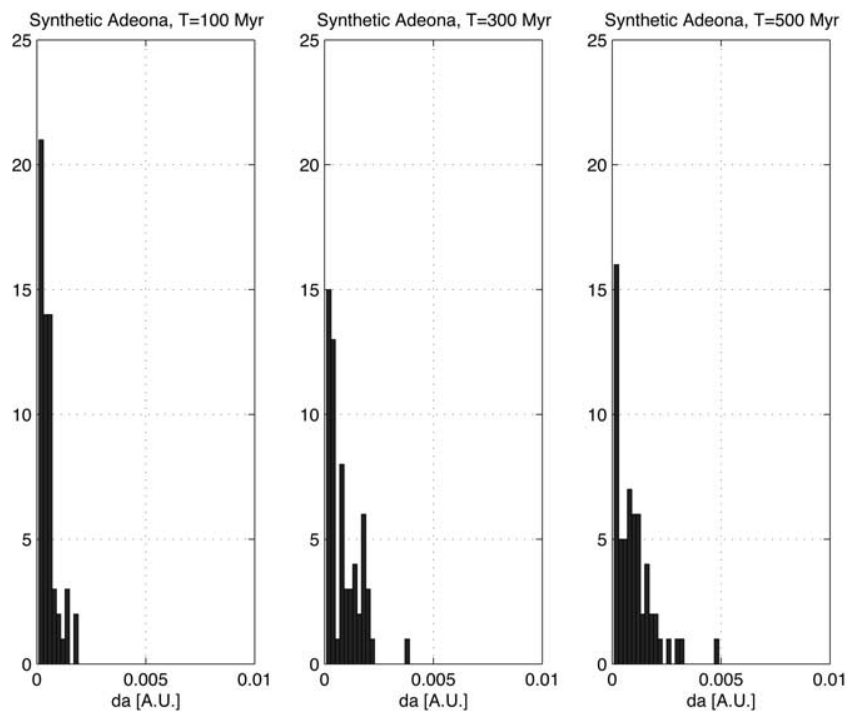

B
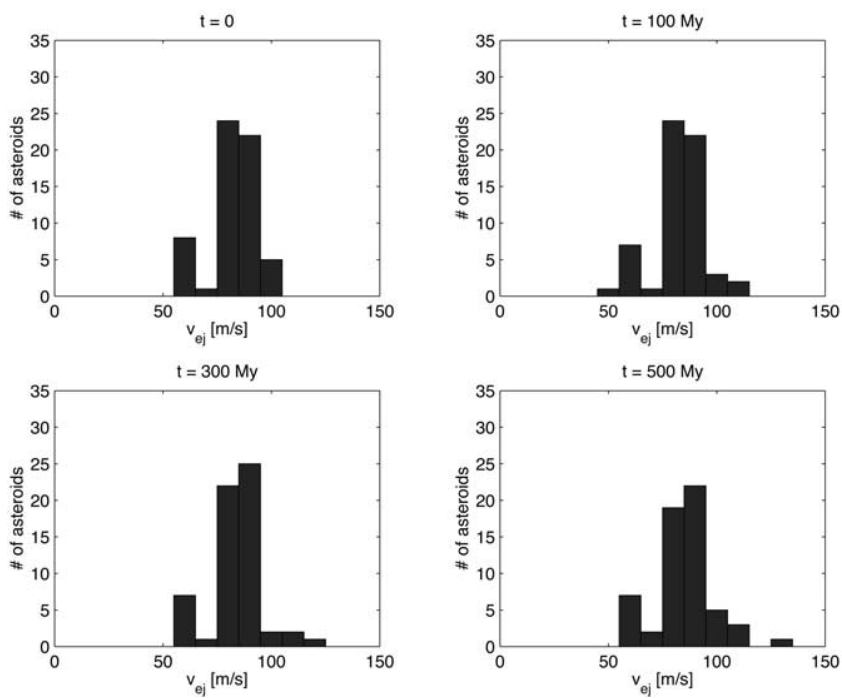

Fig. 5. Histograms of (a) changes in the semimajor axis and (b) apparent ejection velocities at $t=0,100,300$, and $500 \mathrm{Myr}$ for the simulations with the synthetic Adeona family perturbed by the giant planets and the four largest asteroids. We used a bin size of $a=0.0002$ AU.

the parent body (as from Cellino et al., 1999), and $D_{\mathrm{i}}$ is the diameter of the $i$ th fragment. The ejection velocities are taken to be the quadratic sum of velocities at infinity and escape velocities. We distinguish between initial ejection velocities, which are those computed just after the break-up of the parent body, and those "inferred," which are computed at later times, when the asteroids have diffused. ${ }^{4}$

\footnotetext{
${ }^{4}$ We determined the center of mass of the synthetic family by using the averaged elements at $t=0$ and the estimated masses of the family members. We assumed that all objects have the same density $\left(1300 \mathrm{~kg} / \mathrm{m}^{3}\right.$ for C-type and $2500 \mathrm{~kg} / \mathrm{m}^{3}$ for S-type families) and a value of their albedo typical of their spectral type.
} 
A
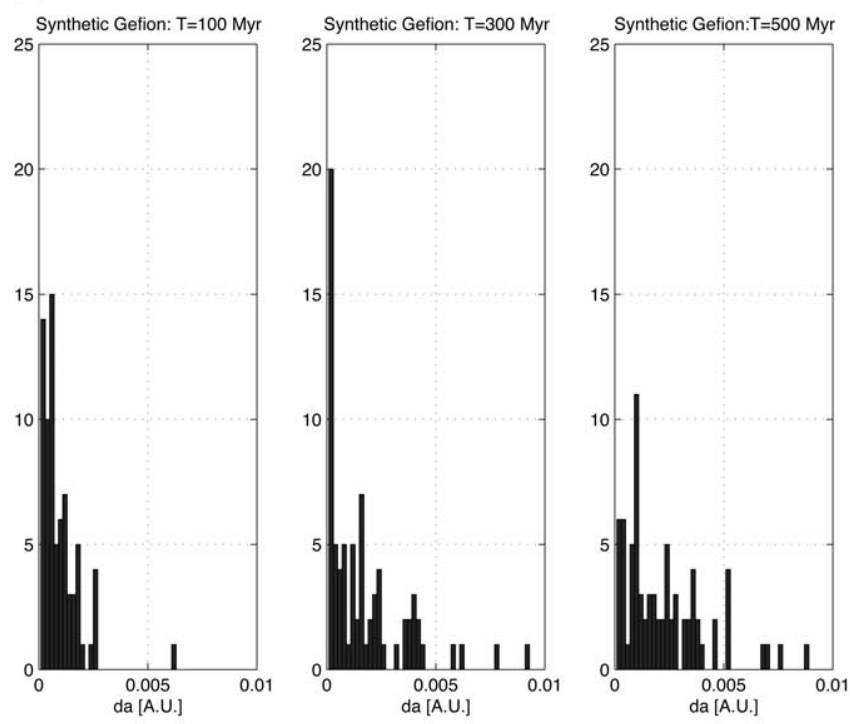

B
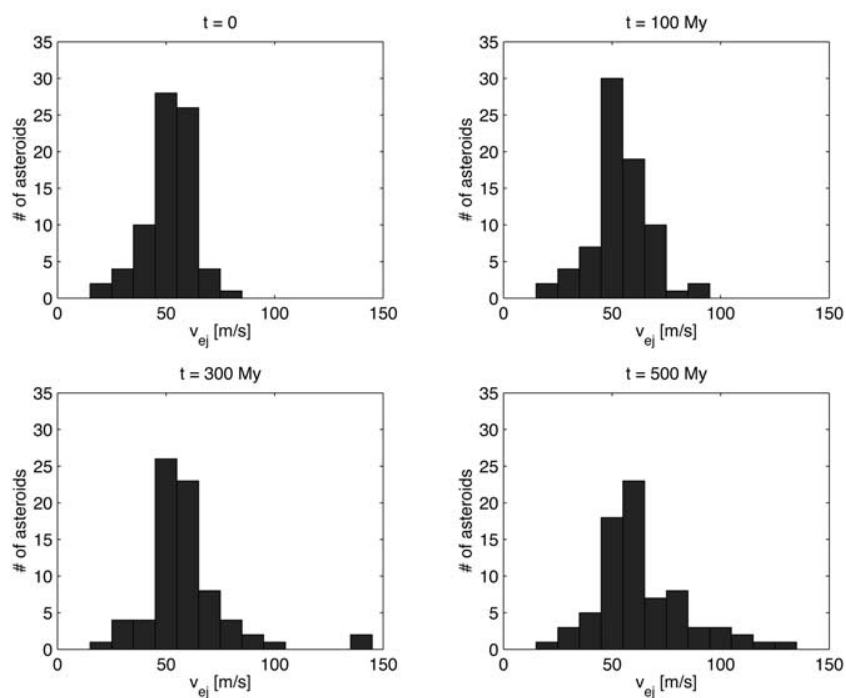

Fig. 6. Histograms of (a) changes in the semimajor axis and (b) apparent ejection velocities at $t=0,100,300$, and $500 \mathrm{Myr}$ for the simulations with the synthetic Gefion family perturbed by the giant planets and the four largest asteroids. We used a bin size of $a=0.0002$ AU.

The inferred velocities so found are functions of $a, e$, and $i$, such that changes in $v_{\mathrm{ej}}$ are not only due to possible close encounters with massive asteroids but also to the interaction of the particle with mean motion or three-body resonances, which affect $e$ and $i$, but not $a$. To avoid overestimating the effect of resonant captures and to include objects whose changes in $e$ and $i$ made them unrecognizable as family members, we eliminated all asteroids whose eccentricity and inclination changed by more than $3 \sigma$ above or below the current mean value for the family. Still, changes in inferred $v_{\text {ej }}$ represent the effect of close encounters (changes in $a, e$, and $i$ ) and resonances (changes in $e$ and $i$ ). The fractional change in inferred ejection velocity due to semi- major axis mobility can be estimated by using the assumption of isotropic ejection velocity fields (i.e., the transversal, the radial, and the normal components of the velocity vectors are all equal). With this assumption, the velocity at infinity is given to first order in $e$ by (Cellino et al., 1999)

$$
v_{\infty}=\frac{\sqrt{3} n \Delta a}{2} \text {. }
$$

A useful rule of thumb to determine the fraction of change in inferred ejection velocity due to semimajor axis mobility is that a $\Delta a$ of 0.01 AU corresponds to a change in $v_{\infty}$ of $55 \mathrm{~m} / \mathrm{s}$ at $a=2.8 \mathrm{AU}$. Changes in $v_{\infty}$ (and therefore in the inferred ejection velocity, if $v_{\text {esc }}$ are much smaller than $\Delta v_{\infty}$ ) are a linear functions of changes in $a$.

Distributions in $v_{\mathrm{ej}}$ at $t=0,100,300$, and $500 \mathrm{Myr}$ are also shown in Figs. $5 \mathrm{~b}$ and $6 \mathrm{~b}$.

At the end of the integration $(t=500 \mathrm{Myr})$ none of the particles in the synthetic Adeona integration had drifted by more than 0.005 AU. Conversely, the real family members showed five particles that drifted by that amount or more. The average drift rates were $5 \times 10^{-4} \mathrm{AU} / 100 \mathrm{Myr}$ in the first $100 \mathrm{Myr}$, in agreement with what was found by Nesvorný et al. (2002). The distribution in ejection velocities was not significantly altered, with the exception of six particles (11\% of the population) that had their "inferred" ejection velocities increased beyond the maximum initial value of $100 \mathrm{~m} / \mathrm{s}$ by the end of the simulation.

The situation was different for the case of the Gefion family. In this case, 8 particles drifted by more than 0.005 AU at $t=500 \mathrm{Myr}$ (16 for the integration of the real Gefion family members). The average drift rates were $10^{-3} \mathrm{AU} /$ $100 \mathrm{Myr}$, i.e., more than twice the drift rate found by Nesvorný et al. (2002) for their integrations of asteroids with $a>2.5$ AU. The inferred ejection velocity distribution for the synthetic family was also considerably modified. At the beginning of the simulation, no particle had an initial ejection velocity larger than $81 \mathrm{~m} / \mathrm{s}$. By the end there were 17 objects with velocities larger than that amount (i.e., $17 \%$ of the 100 particles had their inferred ejection velocity increased beyond the maximum initial value).

Can this displacement be found in the current inferred ejection velocity distribution of the Adeona and Gefion families? Although we cannot precisely determine the past dynamical history of the family members, we can check whether any known large asteroid has a value of $v_{\text {ej }}$ larger than $100 \mathrm{~m} / \mathrm{s}$, which is the maximum ejection velocity generated by the hydro-code of Michel et al. (2001) for such bodies in the Koronis family. To make this test, we used $D$ $>15 \mathrm{~km}$ asteroids, roughly the diameter at which mobility due to close encounters begins to prevail over the mobility due to the Yarkovsky effect for Adeona family members ( $\simeq 10 \mathrm{~km}$ for the Gefion family), assuming a rotation period of $5 \mathrm{~h}$ and thermal properties typical of the spectral types of the two families (Bottke et al. 2001, 2002).

In order to identify $D>15 \mathrm{~km}$ asteroids with high 
Table 1

Members of the Adeona and Gefion families with diameters larger than $15 \mathrm{~km}$ and $v_{\mathrm{ej}}>100 \mathrm{~m} / \mathrm{s}$

\begin{tabular}{|c|c|c|c|c|c|c|c|}
\hline \multicolumn{4}{|c|}{ Adeona } & \multicolumn{4}{|l|}{ Gefion } \\
\hline Ast. \# & $\begin{array}{l}\text { Diameter } \\
(\mathrm{km})\end{array}$ & $\begin{array}{l}\text { Ejection velocities } \\
(\mathrm{m} / \mathrm{s})\end{array}$ & $\begin{array}{l}\text { Spectral } \\
\text { type }\end{array}$ & Ast. \# & $\begin{array}{l}\text { Diameter } \\
(\mathrm{km})\end{array}$ & $\begin{array}{l}\text { Ejection velocities } \\
(\mathrm{m} / \mathrm{s})\end{array}$ & $\begin{array}{l}\text { Spectral } \\
\text { type }\end{array}$ \\
\hline 2333 & 20 & 125 & & 3724 & 22 & 180 & \\
\hline 4157 & 20 & 105 & $\mathrm{Ch}$ & 2386 & 20 & 110 & $\mathrm{~S}$ \\
\hline 3445 & 19 & 105 & & 2521 & 20 & 140 & $\mathrm{~S}$ \\
\hline 46644 & 19 & 110 & & 2053 & 20 & 145 & $\mathrm{~S}$ \\
\hline 997 & 18 & 145 & $\mathrm{Ch}$ & 2905 & 19 & 120 & $\mathrm{~S}$ \\
\hline 1994 & 18 & 135 & & 1433 & 19 & 200 & $\mathrm{~S}$ \\
\hline 19133 & 17 & 165 & & 3860 & 17 & 185 & $\mathrm{~S}$ \\
\hline 5270 & 17 & 355 & & 2157 & 17 & 105 & $\mathrm{~S}$ \\
\hline 5831 & 17 & 105 & & 3910 & 17 & 100 & $\mathrm{~S}$ \\
\hline 17284 & 17 & 320 & & 2595 & 17 & 190 & \\
\hline 22053 & 16 & 110 & & 4417 & 17 & 305 & $\mathrm{~S}$ \\
\hline 13856 & 16 & 215 & & 10064 & 16 & 355 & \\
\hline 36648 & 16 & 250 & & 1839 & 16 & 155 & $\mathrm{~S}$ \\
\hline 7156 & 16 & 110 & & 2875 & 15 & 190 & $\mathrm{~S}$ \\
\hline 4523 & 15 & 135 & $\mathrm{Ch}$ & & & & \\
\hline 15445 & 15 & 130 & & & & & \\
\hline
\end{tabular}

Note. We list the asteroid number, its effective diameter, ejection velocity, and spectral type (Bus and Binzel, 2002), where available.

inferred ejection velocities, we need first to estimate their starting location (i.e., the approximate $(a, e, i)$ orbit of each family's parent body). To zeroth order, it is reasonable to assume that the parent body was initially located at the "center" of each family. We must be careful, though, since $D<15 \mathrm{~km}$ asteroids have been displaced enough by the Yarkovsky effect that some (i) may have escaped the main belt via a mean-motion or secular resonance or (ii) may no longer be recognized as family members. For this reason, we adopted the criterion of defining the family's center as the center of mass of all objects with $H<12.5$ (roughly $D$ $>10 \mathrm{~km}$ ). We believe this eliminates most of the drawbacks discussed above while preserving statistically significant samples.

By using this procedure and Gauss' equations, with the values of the mean anomaly and argument of pericenter of the parent body at the moment of break-up as from Morbidelli et al. (1995), we computed the inferred ejection velocities for members of the Gefion and Adeona families, as determined with Nesvorný's program (see Section 2) and a cutoff in the metric of $80 \mathrm{~m} / \mathrm{s}$. Our goal was to identify members of the Adeona and Gefion families with $v_{\mathrm{ej}}>100$ $\mathrm{m} / \mathrm{s}$ because these asteroids could be good candidates to have experienced past close encounters with large asteroids in the past. Table 1 lists such bodies for the Adeona and Gefion families. The limiting value of $v_{\mathrm{ej}}>100 \mathrm{~m} / \mathrm{s}$ ("critical velocity") must be taken with some caution. While results of hydro-code and $\mathrm{N}$-body simulations for the Koronis and other families (e.g., Michel et al., 2001) do not show any object greater than $15 \mathrm{~km}$ with an ejection velocity larger than this critical value, we recognize that a catastrophic impact can, in theory, produce larger initial velocities for a few of the largest members. However, we believe that this critical value can still be used as a first-order criterion to discriminate bodies with possible past histories of close encounters with massive asteroids or interaction with mean-motion resonances for these two families. Initial ejection velocities for the Adeona and Gefion families should not be larger than the corresponding values found by Michel et al. (2001) for the case of Koronis, since these two families have a higher $M_{\mathrm{LR}} / M_{\mathrm{PB}}$ ratio than Koronis $(0.51$ for Adeona, 0.06 for Gefion, and 0.04 for Koronis, Cellino et al., 1999).

We identified 16 objects in the Adeona family with inferred $v_{\mathrm{ej}}>100 \mathrm{~m} / \mathrm{s}$ and $D>15 \mathrm{~km}$. Three of these objects have known spectral types (Ch) that are consistent with other family members (Bus 1999). This represents $8 \%$ of the Adeona members with $D>15 \mathrm{~km}$ (if we consider the remaining 13 objects with no known spectral type, this corresponds to $36 \%$ of the largest Adeona members), in close agreement with the results of the simulation with synthetic members, in which $11 \%$ of the test particles had their inferred ejection velocities increased beyond the maximum initial value of $100 \mathrm{~m} / \mathrm{s}$.

Fourteen objects that satisfy the same requirements were identified as part of the Gefion family, 11 with the same spectral type as the family (S), and 3 with no known spectral type. This corresponds to $52 \%$ (66\% if we include the objects with no known spectral type) of the $D>15 \mathrm{~km}$ asteroid population in Gefion. Results of the simulation with synthetic members showed that only $17 \%$ of the objects were displaced beyond the maximum initial ejection velocity $(81 \mathrm{~m} / \mathrm{s})$. The discrepancy between observation and results of simulations can be due to (i) the value of critical ejection velocity we used $(100 \mathrm{~m} / \mathrm{s})$, or (ii) the age of the family. The low value of the mass ratio of its largest remnant over the parent body (0.06, Cellino et al., 1999) suggests that the Gefion family is the result of a catastrophic 
disruption, so it is possible that the maximum ejection velocity can be higher in this case (Michel et al., 2001). Moreover, according to our estimates, Gefion should be older than the extent of our integrations (500 Myr), and this could also account for some of the difference we observe.

We believe that those large inferred ejection velocities could possibly be explained in terms of a past history of close encounters with the massive asteroids or interaction with mean-motion resonances. It would be interesting to obtain the spectral types of the remaining asteroids with large velocities in the Adeona and Gefion families to verify whether they are actual members of these two families.

\section{Semimajor axis mobility due to the Yarkovsky effect}

Our integrations showed that close encounters with massive asteroids can modify the inferred ejection velocities of Adeona and Gefion members by at most $60 \mathrm{~m} / \mathrm{s}$ over 500 Myr. While significant, this mechanism still cannot explain the discrepancy observed between the ejection velocities inferred for smaller asteroids and the results of hydro-code simulations. We turn again to the results of Michel et al. (2001) for the Koronis family, which show no objects with $5<D<20 \mathrm{~km}$ having initial ejection velocities larger than $150 \mathrm{~m} / \mathrm{s}$, while most of the real members of the family in that size range have inferred ejection velocities of up to 400 $\mathrm{m} / \mathrm{s}$. (The limit of $400 \mathrm{~m} / \mathrm{s}$ is due to the fact that Koronis is bounded by the $5 / 2$ and $7 / 3$ mean-motion resonances with Jupiter. Objects injected into these resonances, i.e., asteroids with values of $v_{\mathrm{ej}} \simeq 400 \mathrm{~m} / \mathrm{s}$, would have had their $e$ and $i$ significantly increased and would no longer be recognizable as members of the family.) By extrapolating the maximum drift rates found for the Adeona and Gefion familes, we can predict that close encounters can in theory produce a difference in inferred ejection velocity of $250 \mathrm{~m} / \mathrm{s}$ over $2 \mathrm{Gyr}$, the estimated age of Koronis (Marzari et al., 1995), but only for a few objects ( $\simeq 15 \%$ at most), not for the majority of the family members.

To resolve this discrepancy, Bottke et al. (2001) showed that the smaller members of the Koronis family could have been moved predominantly by the nongravitational Yarkovsky force, rather than by close encounters with large asteroids. This scenario potentially explains why some families are sharply bounded by adjacent Kirkwood gaps, why they have asymmetric distributions in $(a, e, i)$ space, and why some family members are present on short-lived orbits. In their tests, Bottke et al. (2001) found that multikilometer asteroids that started close to the center of the Koronis family could be driven by the Yarkovsky effect over hundreds of megayears into several nearby mean-motion and secular resonances. The interaction between these resonances and the Yarkovsky effect allowed many objects to achieve $(a, e, i)$ orbits consistent with observed family members, while those entering the powerful $5 / 2$ or $7 / 3$ mean-motion resonances with Jupiter had their $e$ values pumped up enough to escape the main belt.

We wish to determine whether this same mechanism can explain the orbital distribution of the Adeona and Gefion families (Carruba et al., 2001, 2002). To this end, we performed numerical tests with particles simulating the initial conditions following break-up of the Adeona and Gefion families. The orbital elements and sizes of the synthetic families are those we used previously. Once again, to compare our results with observed family members proper $(a, e$, $i$ ), we averaged the orbital elements derived from our numerical integrations over a running window of $10 \mathrm{Myr}$ and a shift of $10^{5}$ years.

We tracked our test asteroids using the SWIFT-RMVSY integrator (Brož, 1999), which incorporates the diurnal and seasonal Yarkovsky terms from the analytic linearized solutions (Vokrouhlický and Farinella, 1998) into the SWIFTRMVS3 integrator of Levison and Duncan as gravitational perturbations of velocities (Mikkola, 1998). The Yarkovsky effect violates the exact symplecticity of the integrator, but the degree of dissipation is small enough so that this integrator satisfies all test-bed checks (like reproducing the analytical drifts of the semimajor axis on circular orbits, Brož, 1999). Note that Spitale and Greenberg (2001) have shown that the analytic estimates of the semimajor axis mobility are accurate within a few percent, if the orbital eccentricity is not very large $(<0.5)$.

Nondisruptive impacts transmit angular momentum, thereby affecting the orientation of the spin axes, which in turn may change the magnitude and even the direction of the Yarkovsky drift. Our results below will suggest that both asteroid families are less than $850 \mathrm{Myr}$ old. Thus, assuming that the reorientation time (in Myr) is expressed by

$$
\tau_{\text {rot }}=15 \cdot \sqrt{\frac{r}{1 \mathrm{~m}}},
$$

where $r$ is the radius of the asteroid in meters (Farinella et al., 1998; Bottke et al., 2002), on average a body with a radius larger than $\sim 3 \mathrm{~km}$ would not be reoriented since the family formed. Only asteroids smaller than $\sim 0.75 \mathrm{~km}$ in radius would have their spin axes modified more than once during the age of the families. According to these reorientation times, we can neglect nondisruptive impacts. With these assumptions, the standard deviation of changes in the semimajor axis of a cluster of bodies increases linearly with time.

Several parameters determine the Yarkovsky force. We take the asteroids to have random spin-axis orientations, with a rotation period chosen from a Maxwellian distribution peaked at $8 \mathrm{~h}$ and truncated at minimal and maximal periods of 4 and $12 \mathrm{~h}$, respectively (Binzel et al., 1989). The S-type family (Gefion) was assumed to have a regolith with a low thermal conductivity $K=0.001 \mathrm{~W} / \mathrm{m} \mathrm{K}$ (the thermal capacity is $680 \mathrm{~J} / \mathrm{kg} \mathrm{K}$ and the surface density is 1500 $\mathrm{kg} / \mathrm{m}^{3}$ ). These values match reasonably well those of the 
A



B

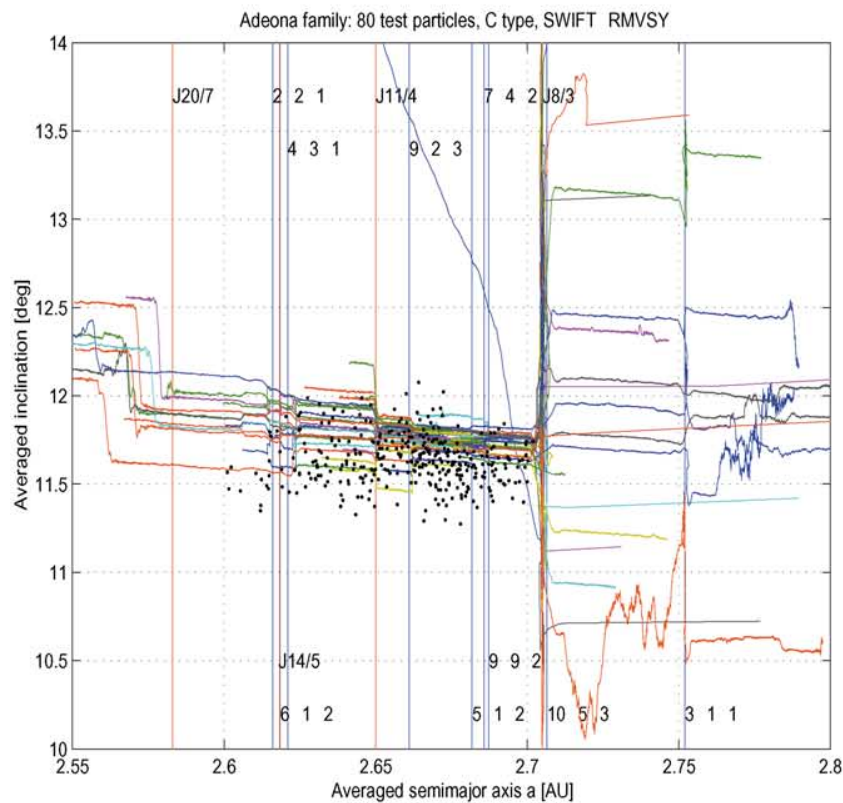

Fig. 7. Histories of (a) averaged $e$ vs averaged $a$ and (b) averaged $i$ vs averaged $a$ for the Adeona family under the action of the Yarkovsky force. The integration lasted $850 \mathrm{Myr}$. Vertical lines indicate the location of mean-motion resonances (red), three-body resonances (blue), and the center of the $g-g_{6}+g_{5}$ secular resonance (yellow) computed for $i=11.6^{\circ}$; the specific resonances are identified on the top and bottom axes of the figures. Black dots locate family members as determined with a HCM method by Nesvorný. The colored lines represents the evolution in time of the test particles: prograde-rotating particles evolved toward larger values of $a$ and interacted with the 8/3 mean-motion resonance, which is also the boundary of the Adeona family, while retrograde-rotating particles evolved toward the 2-2-1, 4-3-1, and 6-1-2 three-body resonances where they were subsequently scattered in $e$ and $i$.
A

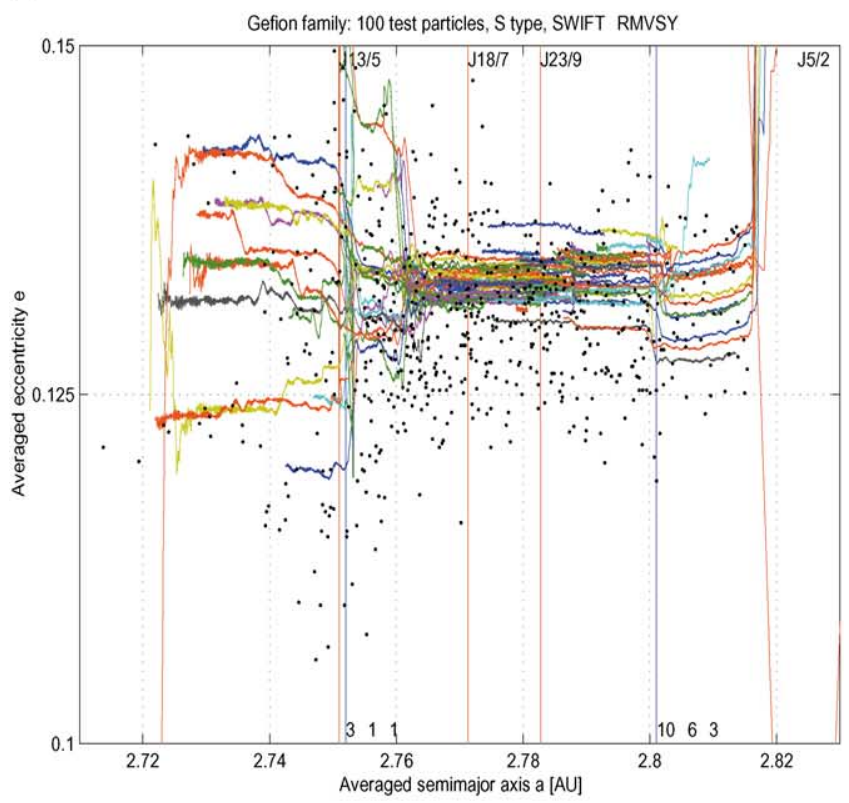

B

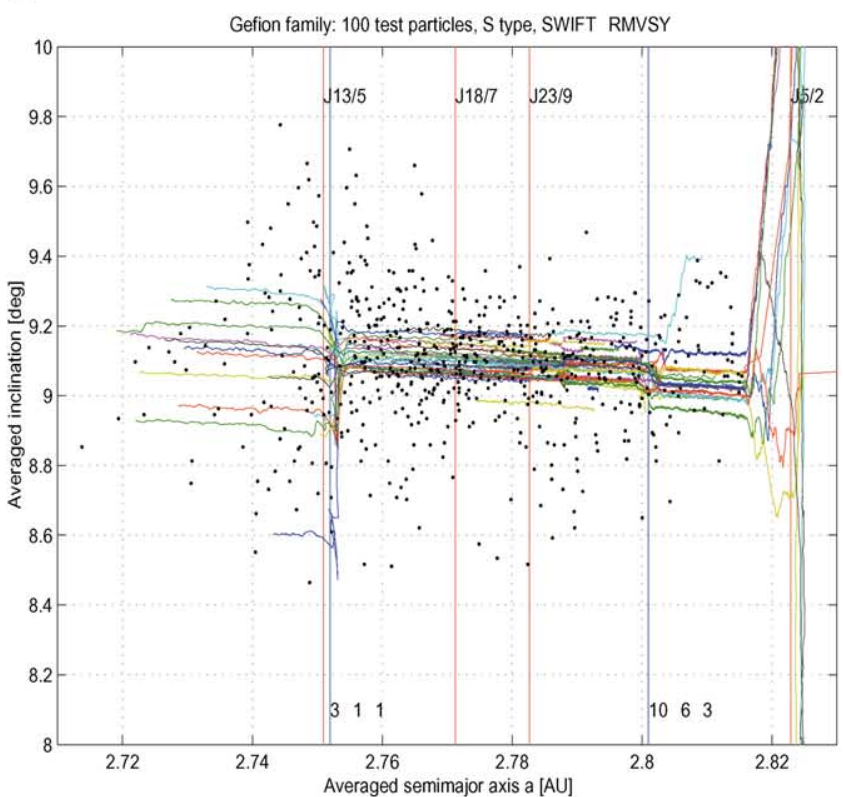

Fig. 9. Histories of (a) averaged $e$ vs averaged $a$ and (b) averaged $i$ vs averaged $a$ for the Gefion family. The integration lasted $750 \mathrm{Myr}$. Vertical lines indicate the location of mean-motion resonances (red), and three-body resonances (blue). Black dots locate members of the family as determined with a HCM method by Nesvorný. The colored lines represent the evolution in time of the test particles: prograde-rotating particles evolved toward larger values of $a$ and interacted with the $5 / 2$ mean-motion resonance, which is also the boundary of the Gefion family, while retrograde-rotating particles evolved toward the 13/5 mean-motion and 3-1-1 three-body resonance and were subsequently scattered in $e$ and $i$. This is consistent with the observed distribution of actual Gefion members as determined by Nesvorný's code. 

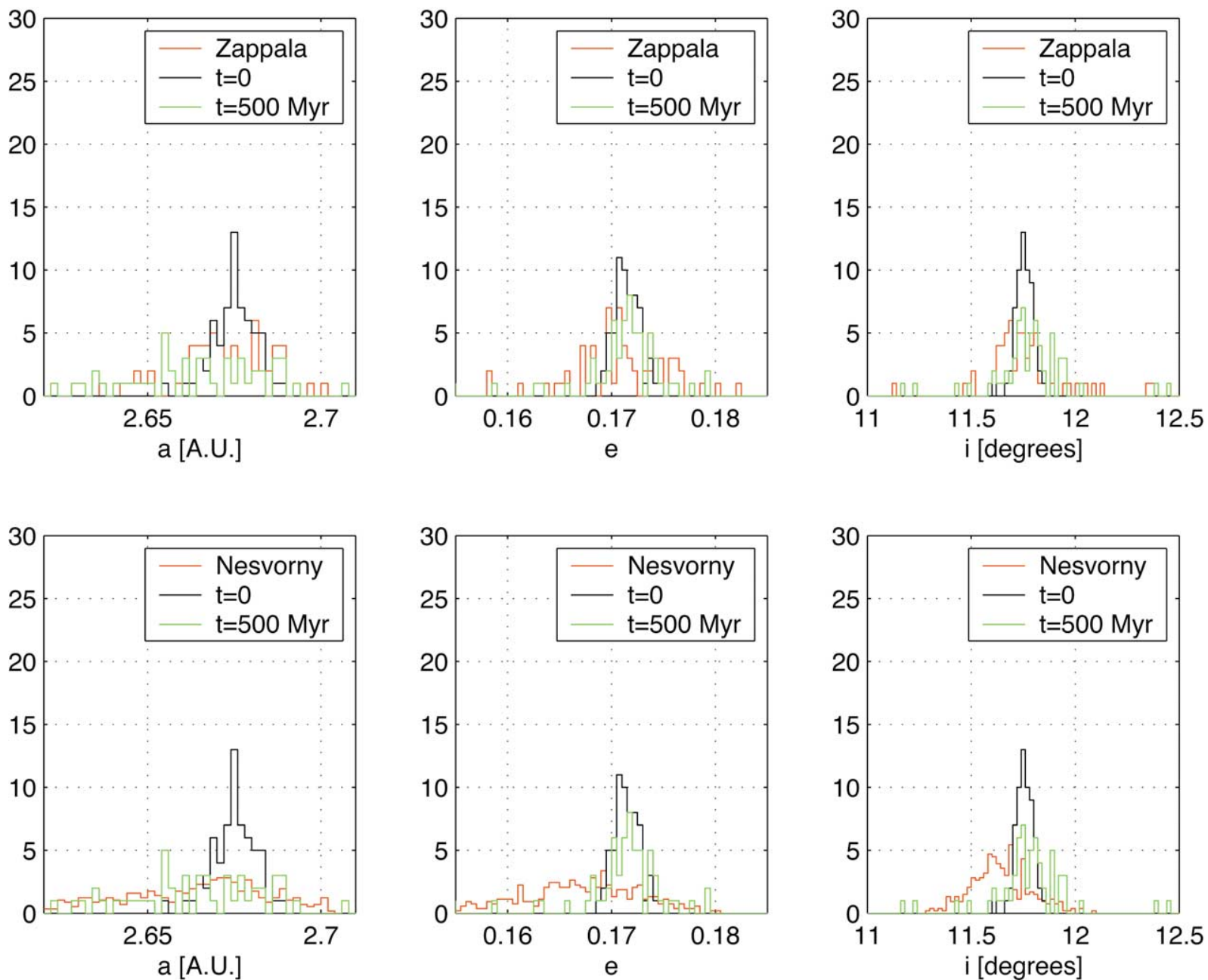

Fig. 8. The Adeona family's distribution in averaged $a$, $e$, and $i$. We compared these for our test particles at $t=0$ and 500 Myr against those from two schemes. In the first row we display the distribution of family members from Zappalás (Zappalá et al., 1995) files, while in the second row we show the distribution of family members determined by Nesvorný. There is an offset in the distribution in $e$ and $i$ between the two distributions.

surfaces of the Moon (Rubincam, 1995), and are consistent with observations of small asteroids by ISO (Lagerros et al., 1999). We also chose the Bond albedo to be 0.1 , a thermal emissivity of 0.95 , and a bulk density of $2500 \mathrm{~kg} / \mathrm{m}^{3}$, typical of S-type, which is consistent with the recent determinations of mean bulk density of S-type asteroids 243 Ida, 433 Gaspra, and 433 Eros from space missions (Thomas et al., 1996).

For the C-type family (Adeona, Ch type) we assumed that the surface conductivity was $0.01 \mathrm{~W} / \mathrm{m} \mathrm{K}$; this somewhat higher choice might result from ice components in the regolith. Direct observational evidence is virtually absent in this case, with the exception of models of cometary activity. We may therefore admit somewhat larger uncertainties in modeling the Yarkovsky effect for the C-type families. We used a Bond albedo of 0.05 and thermal emissivity of 0.95 . The assumed lower value of the bulk density, $1300 \mathrm{~kg} / \mathrm{m}^{3}$, should follow from icy components and high porosity.

Our simulations for the Adeona family are shown in Fig. 7. The Adeona family is bounded by the $8 / 3$ mean-motion resonance with Jupiter, and a trio of three-body resonances (asteroid, Jupiter, and Saturn: the 2-2-1, 4-3-1, and 6-1-2), with only 12 members beyond the 2-2-1 resonance at 2.616 AU. The family is also crossed by the $11 / 4$ resonance and by other three-body resonances.

The test particles started to drift inward or outward according to the direction of their spins (retrograde and prograde, respectively, if the diurnal version of the Yarkovsky effect was larger than the seasonal one). Once they reached the $8 / 3$ resonance, they were scattered in eccentricity and could no longer be recognized as family members. Thus, we suspect that various members of the real Adeona family have similarly drifted across this resonance, and now lie in the region between the 8/3 and 3-1-1 resonances.

Other test particles interacted with the $g-2 g_{6}-g_{5}$ secular resonance and experienced considerable changes in $e$, but not in $i$. In our simulations, we chose conservative initial conditions (see Appendix), and we centered the synthetic Adeona family in the midst of the observed family according to Cellino et al. (1999); this is offset in $e$ and $i$ 
with respect to the family computed by us (see Fig. 8 with distributions in $a, e$, and $i$ for $t=0,500 \mathrm{Myr}$, the distribution for our members was normalized to the number of test particles used). As a result, we were not able to match the distributions in $e$ and $i$. We plan to conduct new simulations with more appropriate initial conditions.

The history of the Gefion family is shown in Fig. 9. Gefion is crossed by the $13 / 5,18 / 7$, and $23 / 9$ mean-motion resonances with Jupiter and is bounded by the $5 / 2$ resonance at $2.82 \mathrm{AU}$. The family is strongly dispersed in $e$ by the $13 / 5$ resonance and (possibly) by an unindentified higher-order secular resonance at $a \simeq 2.76 \mathrm{AU}$. The interactions of Yarkovsky $a$ diffusion with the 3-1-1 and 13/5 resonances seem to give a plausible explanation for the peaked distribution in the $e-a$ and $i-a$ planes at $\simeq 2.75 \mathrm{AU}$, which would otherwise require a quite asymmetrical ejection velocity field. Once particles reached the $5 / 2$ resonance they were scattered in eccentricity and were no longer identifiable as family members. This could explain why the Gefion family appears to be bounded by the $5 / 2$ resonance.

Also in this case our conservative choice of initial conditions and the fact that we centered the simulated family in the midst of Zappalá's family caused a mismatch in the simulated distributions in $e$ and $i$.

\section{Ages of the families}

We estimated the ages of the Adeona and Gefion families from our simulations using two different methods. The first is based on the dispersions in $e$ and $i$ of the real family members integrated under the effect of close encounters with large asteroids (Nesvorný et al., 2002), while the second compares the current observed values of $\sigma_{a}$ with the results of our Yarkovsky simulations. Both methods are subject to large uncertainties, so any estimate must be taken with caution.

In the first method we consider only real family members larger than $15 \mathrm{~km}$, so as to limit any mobility due to the Yarkovsky effect. We compute the dispersions in $e$ and $i$ resulting from chaotic diffusion by mean-motion and threebody resonances (which, in $\simeq 5 \%$ of the cases, was helped by close encounters with massive asteroids that injected asteroids into resonances). In our runs, only 9 members of Adeona and 8 of Gefion had diameters larger than $15 \mathrm{~km}$, such that our results may be affected by errors due to small-numbers statistics.

We assumed that:

(i) $\sigma_{e}$ and $\sigma_{i}$ are proportional to $t^{1 / 2}$, with a constant of proportionality dependent on the current value of dispersion in $e$ and $i$; i.e., we assumed that chaotic diffusion in meanmotion resonances can be modeled by a random walk (Murray and Holman (1997) note that $\sigma_{e}$ and $\sigma_{i}$ follow a time dependency different than that of $\sigma_{a}$ ); and

(ii) the initial distributions in $e$ and $i$ and $\delta$ functions: this unrealistic assumption sets an upper limit on the family age.
Table 2

Estimates of the ages of the Adeona and Gefion families

\begin{tabular}{lllll}
\hline Integrator & SyMBA & SyMBA & SWIFT-RMVSY & $\begin{array}{l}\text { SWIFT-RMVSY } \\
\end{array}$ \\
& $T_{e}(\mathrm{Myr})$ & $T_{i}(\mathrm{Myr})$ & $\begin{array}{l}D_{\text {cutoff }}=80 \mathrm{~m} / \mathrm{s} \\
T_{a}(\mathrm{Myr})\end{array}$ & $\begin{array}{l}D_{\text {cutoff }}=90 \mathrm{~m} / \mathrm{s} \\
T_{a}(\mathrm{Myr})\end{array}$ \\
\hline Adeona & $250 \pm 240$ & $750 \pm 450$ & $<430$ & $<580$ \\
Gefion & $340 \pm 390$ & $490 \pm 360$ & $<750$ & $>750$
\end{tabular}

Note. The second and third columns show the results obtained from the time evolution of $\sigma_{e}$ and $\sigma_{i}$ of members with $D>15 \mathrm{~km}$ (SyMBA integrations). Errors correspond to $3 \sigma$ values of $T(t)$ (see Eq. (11)). The last two columns provide estimates from the time evolution of $\sigma_{a}$ according to the Yarkovsky integrations and the values of $\sigma_{0}$ for members of the families from Nesvorný's program with cutoffs of 80 and $90 \mathrm{~m} / \mathrm{s}$ (see also Fig. 11).

We then calculated $\sigma_{e}$ and $\sigma_{i}$ for each time step of the averaged elements; since at the beginning of the simulation $\sigma_{0}=C \sqrt{T}$, where $T$ is the family age, while at time $t, \sigma_{t}=$ $C \sqrt{t+T}$, it follows that

$$
T(t)=\frac{\sigma_{0}^{2} t}{\sigma_{t}^{2}-\sigma_{0}^{2}}
$$

We computed $T(t)$ for each time step of the averaged elements $\left(10^{5}\right.$ years $)$, and we then averaged over the length of the integration (we ignore the first $100 \mathrm{Myr}$ of data in order to have values of $\sigma_{t}$ significantly different from those of $\sigma_{0}$ ). Table 2 gives our estimates of the family ages, with errors corresponding to the $3 \sigma$ values of $T(t)$. We found ages of $750 \pm 450 \mathrm{Myr}$ for Adeona and of $490 \pm 360 \mathrm{Myr}$ for Gefion. This means that the Adeona family is less than 1200 Myr old, while the Gefion family is less than 850 Myr old.

Next we used the results of our Yarkovsky simulations to match the current values of $\sigma_{a}$ with the dispersion computed in the simulation for the synthetic family's members. Any estimate obtained with this method is uncertain due to the inherent arbitrariness in the choice of initial conditions. In particular, since the Yarkovsky effect acts mainly on the semimajor axis, due to the conservative value of velocity at infinity with which our synthetic families were generated, we were not able to reproduce the distributions in $e$ and $i$. Nevertheless, we can try to estimate the family ages from $\sigma_{a}(t)$. Fig. 11 plots the time evolutions of $\sigma_{a}$ for Adeona and Gefion.

Horizontal lines represent the current values of $\sigma_{a}$ for family members found by Nesvornýs with cutoffs of 80 and $90 \mathrm{~m} / \mathrm{s}$, for asteroids having diameters between 2 and 4 $\mathrm{km}$ (see Section 2). We used this set of diameters because we needed to consider the same size ranges for simulated and observed objects. We believe these size ranges were sampled well enough in both populations. The uncertainty in the dispersion at impact in $a$ can affect the estimate of the families' ages derived with this method. An initial dispersion of the synthetic families smaller than the actual values would result in an estimate of the age older than the real value (and vice versa). Since in our work we used synthetic families considerably more compact in $a$ than Adeona and 

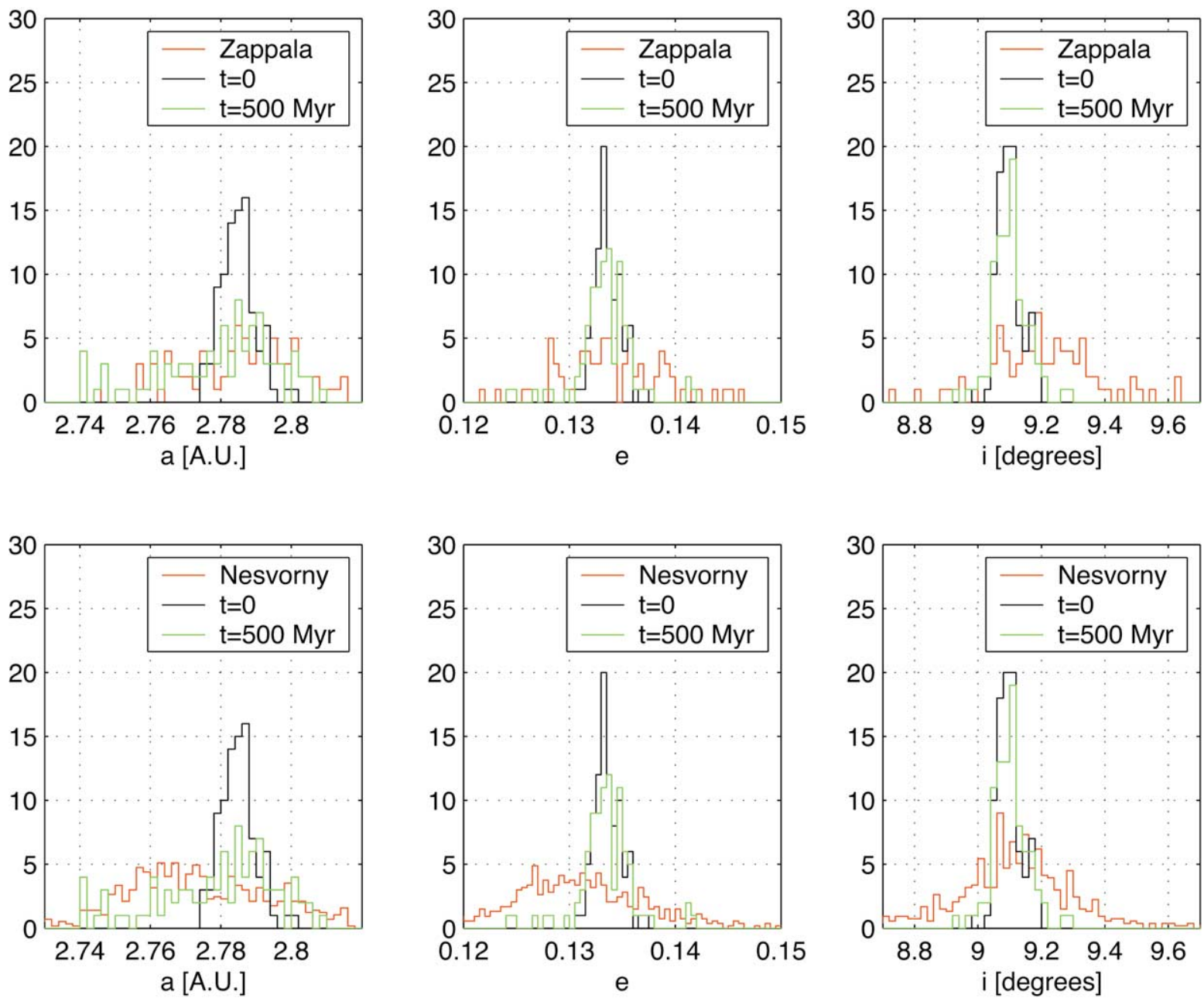

Fig. 10. The Gefion family's distribution in averaged $a$, $e$, and $i$. We computed these for our test particles at $t=0$ and 500 Myr against those from two schemes. In the first row we show the distribution of family members from Zappalá's (Zappalá et al., 1995) files, while the second row shows the distribution of family members determined by Nesvorný. There is an offset in the distribution in $e$ and $i$ between the two distributions.

Gefion were just after the impact (see Appendix), our estimates of the ages should be interpreted as upper limits. We found that Adeona's age is less than $600 \mathrm{Myr}$, while Gefion's age is $750 \mathrm{Myr}$ or more (for $d_{\text {cutoff }}=90 \mathrm{~m} / \mathrm{s}$, see Table 2).

Combining the results of both methods, we estimate that the Adeona family is roughly less than $600 \mathrm{Myr}$ old (the upper limit of $1200 \mathrm{Myr}$ given by the time evolution of $\sigma_{i}$ obtained by the integrations with massive asteroids is not compatible with the results of the Yarkovsky integrations) and the Gefion family is less than 850 Myr old. Because both families have quite clustered proper-element distributions, we believe they can be considered relatively young families, compared with larger families like Koronis and Themis whose ages is estimated to be of $2 \mathrm{Byr}$ or more (Marzari et al., 1995). It is plausible that small families such as these examples did not have enough time to disperse their members' distances beyond the quasi-random level of Zappalà et al. (1995) (i.e., the average distance in term of velocity among main-belt asteroids).
The fact that the ages deduced from both methods are, within errors, in rough agreement suggests that (i) our results are reasonable and (ii) we may be able to use this technique in the future to estimate the ages of other asteroid families. It also provides some support to the idea that small family members are not static in time but instead are dynamically spreading with age.

\section{Summary and conclusions}

We investigated the long-term effects of close encounters with massive asteroids in order to determine the degree to which the semimajor axes (and, more generally, the inferred ejection-velocity field) of asteroid family members can be scattered by this mechanism. We integrated real and synthetic members of two asteroid families (Adeona and Gefion) under the influence of the four giant planets and the four most massive asteroids, which our Monte Carlo simulations suggested as primary perturbers. 

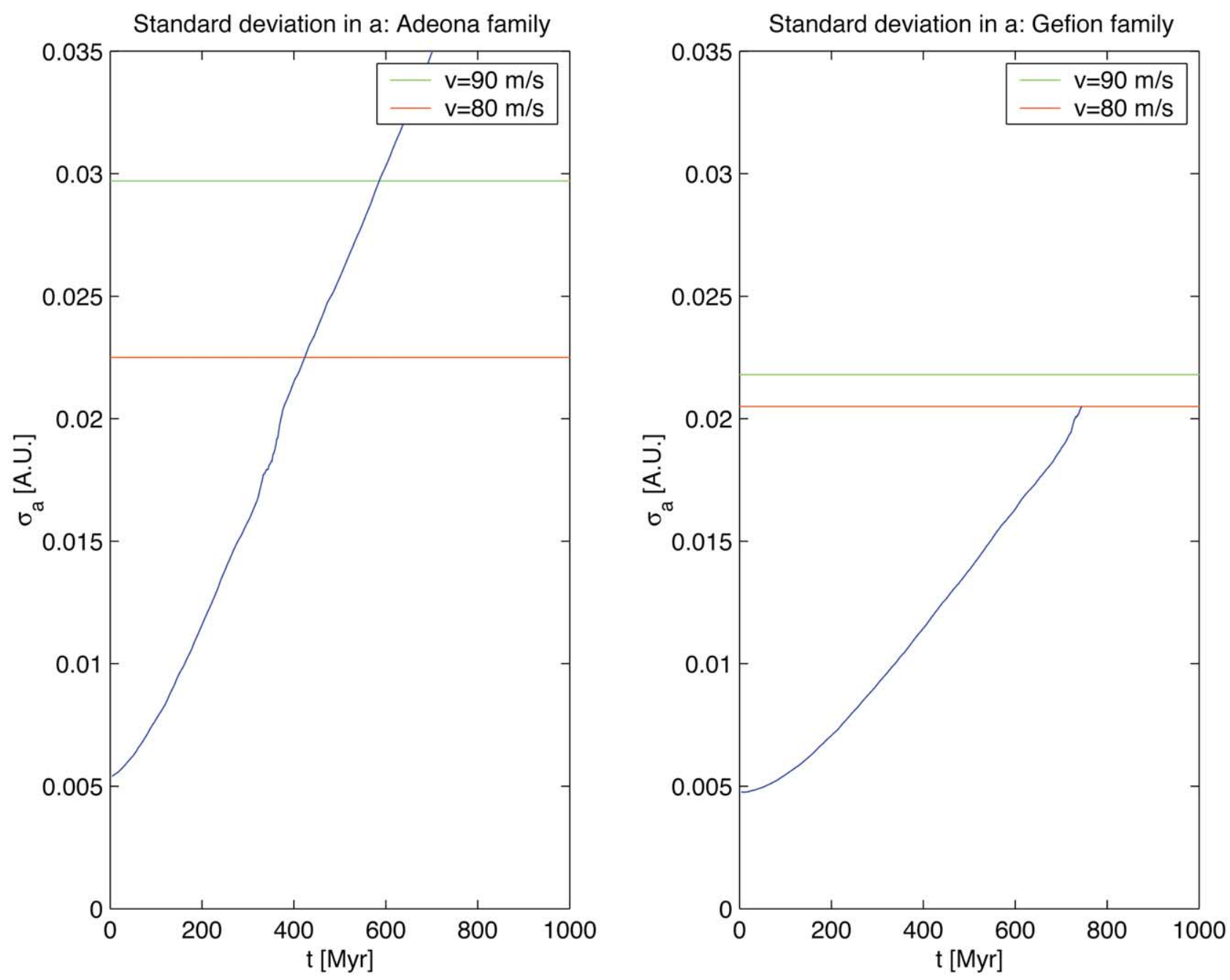

Fig. 11. Time evolution of $\sigma_{a}$ for the Yarkovsky simulations of the Adeona and Gefion families.

We determined the averaged drift rates for the Adeona (5 $\left.\times 10^{-4} \mathrm{AU} / 100 \mathrm{Myr}\right)$ and for the Gefion $\left(10^{-3} \mathrm{AU} / 100\right.$ Myr) families, and computed their time evolution. Like Nesvorný et al. (2002), we found that close encounters disperse the asteroids' semimajor axes with a power-law exponent $B>0.5$, i.e., faster than a random walk law. Unlike previous studies that had an integration time of 120 Myr, our longer integrations ( $\simeq 500 \mathrm{Myr}$ ) show that a simple power law does not fit the change in the standard deviation of the semimajor axis. In particular, $B$ is an evolving (usually decreasing) function of time. We believe this result can be explained as a consequence of the close encounters: once an encounter takes place, most of the test particles go onto orbits having lower collision probabilities with their main perturbers, thereby reducing $B$.

Our integrations show that close encounters with 1 Ceres and the other largest asteroids alone are insufficient to dynamically spread the semimajor axis distribution of family members by more than $4 \%$ of the original value. A possible exception to this rule would be with the Gefion family, the family closest in proper-element space to 1
Ceres, the main perturber. Based on this result, we believe that properties like the family's center can be safely determined by using the largest family members, provided that the sample is statistically significant and that none of these bodies has been previously captured in any mean-motion, three-body, or secular resonance.

This result does not imply that close encounters are totally irrelevant. In our integrations with synthetic families we found that family members can be dispersed enough to increase their inferred ejection velocities. In our tests, $\simeq 10 \%$ of the integrated bodies achieved velocities over 100 $\mathrm{m} / \mathrm{s}$, the maximum velocity for $D>15 \mathrm{~km}$ fragments found by hydro-code and $\mathrm{N}$-body code simulations (e.g., Michel et al., 2001).

We also performed integrations that account for the Yarkovsky effect. We show that, as similarly found by Bottke et al. (2001), Nesvorný et al. (2002), and Vokrouhlický et al. (personal communication, 2002), the Yarkovsky effect seems to explain why families are neatly bounded by resonances (the 8/3 mean-motion and the 2-2-1, 4-3-1, and 6-1-2 three-body resonances for the Adeona family; the 5/2 
and 13/5 mean-motion resonances and the 3-1-1 three-body resonance for the Gefion family), and how the interaction between the Yarkovsky effect and mean-motion resonances can explain asymmetries in the orbital element distribution (the region around the 13/5 resonance for the Gefion family and that around the 11/4 resonance for the Adeona family). Based on our results from both modeling efforts, we estimate that the Adeona and Gefion families are less than 600 Myr and less than 850 Myr old, respectively.

Results of our simulations can be found at http://www. astro.cornell.edu/ valerio/ast_families.html.

\section{Acknowledgments}

The authors thank David Vokrouhlický and Mira Brož for their suggestions and for allowing us to use the SWIFTRMVSY integrator. We are also very grateful to Hal Levison and Martin Duncan for having put SyMBA at our disposal. We gratefully acknowledge the computational resources provided to us for this project by the Cornell Theory Center. We acknowledge Alessandro Morbidelli, Alberto Cellino, and Vincenzo Zappalà for their useful comments and observations. Reviews by Patrick Michel and Richard Greenberg improved our presentation measurably. The first author thanks Elise Furlan and Ömer IIday for their constant support and suggestions. This study has been supported by NASA Grants NAGN-310 and NAG5-11480. We also acknowledge the support of the international travel program of the NSF.

\section{Appendix: generating compact families}

To generate compact families for the simulations, we first computed the radius of the parent body (all quantity were computed in the S.I. system)

$$
R_{\mathrm{PB}}=R_{\mathrm{LR}} \cdot\left(\frac{M_{\mathrm{PB}}}{M_{\mathrm{LR}}}\right)^{1 / 3} \text {, }
$$

where PB stands for "parent body" and LR for "largest remnant." The mass ratio of the largest remnant with respect to the parent body comes from Cellino et al. (1999). We then computed the specific (i.e., per unit mass) energy for the parent body's fragmentation (Benz and Asphaug 1999) from

$$
Q_{D}^{*}=0.4 \cdot \rho_{\mathrm{PB}} \cdot\left(R_{\mathrm{PB}}\right)^{1.36},
$$

where $\rho_{\mathrm{PB}}$ is the bulk density of the parent body. We must point out that this value of $Q_{D}^{*}$ leads to a mass ratio $M_{\mathrm{LR}} /$ $M_{\mathrm{PB}}$ of 0.5 . While this is approximately true for the Adeona family (mass ratio of 0.51 ), it is incorrect for the Gefion family, mass ratio 0.06 (Cellino et al., 1999). In this case the actual $Q_{D}^{*}$ may be higher and the real family can be initially less compact than that simulated here. Since our goal is to generate a synthetic family as compact as possible, we used Eq. (13) also for the case of the Gefion family.

With these assumptions, the kinetic energy of a fragment of mass $m$ is then

$$
T=\frac{1}{2} m v_{\mathrm{ej}}^{2}=m Q_{D}^{*} \frac{f_{\mathrm{KE}}}{2},
$$

where $f_{\mathrm{KE}}$ is an unknown factor that takes into account that only a portion of the specific energy $Q_{D}^{*}$ goes into the fragment's kinetic energy. Fragments leave the parent body with a speed independent of their mass, consistent with experiments (Giblin et al., 1998). The factor $f_{\mathrm{KE}}$ is poorly determined: experiments and hydro-code simulations suggest values between 0.01 and 0.1 . In order to generate families as compact as possible, we chose $f_{\mathrm{KE}}=0.01$.

From Eq. (14) the mean quadratic speed of the fragments is

$$
v_{\mathrm{ej}}^{2}=Q_{D}^{*} f_{\mathrm{KE}} .
$$

The distribution of the ejection speeds is then taken to be Maxwellian. The escape velocity from the parent body is (Petit and Farinella, 1993)

$$
v_{\mathrm{esc}}^{2}=1.64 \frac{G M_{\mathrm{PB}}}{R_{\mathrm{PB}}},
$$

where 1.64 is an empirical factor. Only those fragments with $v_{\mathrm{ej}}>v_{\mathrm{esc}}$ leave the parent body; the others reaccumulate and are not taken into account. The fragments that escape have velocities with respect to the parent body given by

$$
v_{\infty}^{2}=v_{\mathrm{ej}}^{2}-v_{\mathrm{esc}}^{2} .
$$

We thus obtain a distribution $N\left(v_{\infty}\right)$ for the ejected fragments in terms of $v_{\infty}$ (Farinella et al., 1993). Assuming that the ejection is 3-D isotropic, from $N\left(v_{\infty}\right)$ it is possible to deduce the correlated distributions $N\left(v_{\mathrm{T}}\right), N\left(v_{\mathrm{R}}\right)$, and $N\left(v_{\mathrm{W}}\right)$ with respect to the tangential, radial, and vertical velocities. Using Gauss' equations (Zappalà et al., 1995, Morbidelli et al., 1995), from $v_{\mathrm{T}}, v_{\mathrm{R}}$, and $v_{\mathrm{W}}$ we can compute $a$, $e$, and $i$ with respect to the parent body. In order to do that, we need to assume values of $f$ and $\omega$, respectively the mean anomaly and the argument of pericenter of the parent body at the moment of impact. We used the values of these angles from Morbidelli et al. (1995), where available, and otherwise we chose $f=90^{\circ}$ and $f+\omega=45^{\circ}$. Moreover, to obtain that the values of $a, e$, and $i$ computed above represent variations in the space of proper elements, we chose the angles of the fragments to equal the angles of the parent body, at a given epoch. To first order, if all angles are equal, the differences in the values of the osculating $a, e$, and $i$ are equal to the differences in proper $a, e$, and $i$. 


\section{References}

Benz, W., Asphaug, E., 1999. Catastrophic disruption revisited. Icarus 142, 5-20.

Biesiadecki, J.J., Skeel, R.D., 1993. Dangers of multiple time-step methods. J. Comput. Phys. 109, 318-328.

Binzel, R.P., Farinella, P., Zappalà, V., Cellino, A., 1989. Asteroid rotation rates-distribution and statistics, in: Binzel, R.P., Gehrels, T., Matthews, M.S. (Eds.), Asteroids II, Univ. of Arizona Press, Tucson, pp. 416-441.

Bottke Jr., W.F., Greenberg, R., 1993. Asteroidal collision probabilities. Geophys. Res. Lett. 20, 879-881.

Bottke, W.F., Nolan, M.C., Greenberg, R., Kolvoord, R.A., 1994. Velocity distributions among colliding asteroids. Icarus 107, 255-268.

Bottke Jr., W.F., Vokrouhlický, D., Brož, M., Nesvorný, D., Morbidelli, A., 2001. Dynamical spreading of asteroid families via the Yarkovsky effect: the Koronis family and beyond. Science 294, 1693-1696.

Bottke Jr., W.F., Vokrouhlický, D., Rubincam, D., Brož, M., 2003. Dynamical evolution of asteroids and meteoroids using the Yarkovsky effect, in: Bottke, W.F., Cellino, A., Paolicchi, P., Binzel, R.P. (Eds.), Asteroids III, Univ. of Arizona Press, Tucson, 399-408.

Bowell, E., Hapke, B., Domingue, D., Lumme, K., Peltoniemi, J., Harris, A.W., 1989. Application of photometric models to asteroids, in: Binzel, R.P., Gehrels, T., Matthews, M.S. (Eds.), Asteroids II, Univ. of Arizona, Tucson, pp. 524-556.

Brož, M., 1999. Orbital Evolution of Asteroidal Fragments due to Planet Gravitation and Thermal Effects. Thesis, Charles University, Prague, Czech Republic.

Burns, J.A., Lamy, P.L., Soter, S., 1979. Radiation forces on small particles in the Solar System. Icarus 40, 1-48.

Bus, J.S., 2002. Compositional Structure in the Asteroid Belt: Results of a Spectroscopic Survey. Ph.D. Thesis, M.I.T., Cambridge, MA.

Bus, J.S., Binzel, R.P., 2002. Phase 2 of the small Main-Belt asteroid spectroscopic survey. Icarus 158, 146-177.

Carruba, V., Burns, J.A., Bottke, W., Morbidelli, A., 2000. Asteroid mobility due to encounters with Ceres, Vesta, Pallas: Monte Carlo codes versus direct numerical integrations. Bull. Am. Astron. Soc. 32 , 1019.

Carruba, V., Burns, J.A., Bottke, W., Nesvorný, D., Zappalà, V., 2001. Gravitational scattering of asteroid families by massive asteroids. Bull Am. Astron. Soc. 33, 1136.

Carruba, V., Burns, J., Bottke, W., and Nesvorný, D., 2002. Dynamical evolution of the Adeona and Gefion asteroid families, in: Celleti, A., Ferraz-Mello, S., Henrard, J. (Eds.), Modern celestial mechanics: from theory to applications. Kluwer Academic Publishers, pp. 365-368.

Cellino, A., Michel, P., Tanga, P., Zappalà, V., Paolicchi, P., Dell'Oro, A., 1999. The velocity-size relationship for members of asteroid families and implications for the physics of catastrophic collisions. Icarus 141, 79-95.

Chambers, J.E., 1999. A hybrid symplectic integrator that permits close encounters between massive bodies. Mon. Not. R. Astron. Soc. 304 793-799.

Danby, J.M.A., 1988. Fundamentals of Celestial Mechanics, 2nd ed. Willmann-Bell, Richmond, VA.

Dones, L., Gladman, B., Melosh, H.J., Tonks, W.B., Levison, H.F., Duncan, M., 1999. Dynamical lifetimes and final fates of small bodies: orbit integrations vs. Öpik calculations. Icarus 142, 509-524.

Duncan, M.J., Levison, H.F., Lee, M.H., 1998. A multiple time-step symplectic algorithm for integrating close encounters. Astron. J. 116, 2067-2077.

Farinella, P., Davis, D.R., 1992. Collision rates and impact velocities in the main asteroid belt. Icarus 97, 111-123.

Farinella, P., Vokrouhlický, D., 1999. Semimajor axis mobility of asteroidal fragments. Science 283, 1507-1510.
Farinella, P., Gonczi, R., Froeschlé, Ch., Froeschlé, C., 1993. The injection of asteroid fragments into resonances. Icarus 101, 101-174.

Farinella, P., Vokrouhlický, D., Hartmann, W.K., 1998. Meteorite delivery via Yarkovsky effect. Icarus 132, 378-387.

Fujiwara, A., Cerroni, P., Davis, D.R., Ryan, E., DiMartino, M., Holsapple, K., Housen, K., 1989. Experiments and scaling laws on catastrophic collisions, in: Binzel, R.P., Gehrels, T., Matthews, M.S. (Eds.), Asteroids II, Univ. of Arizona Press, Tucson, pp. 240-265.

Giblin, I., Petit, J.M., Farinella, P., 1998. The properties of fragments from catastrophic disruption events. Icarus 134, 77-112.

Gladman, B.J., Migliorini, F., Morbidelli, A., Zappalà, V., Michel, P., Cellino, A., Froeschlé, Ch., Levison, H.F., Bailey, M., Duncan, M., 1997. Dynamical lifetimes of objects injected into asteroid belt resonances. Science 277, 197-201.

Greenberg, R., 1982. Orbital interactions: a new geometrical formalism. Astron. J. 87, 184-195.

Knežević, Z., Milani, A., 2000. Synthetic proper elements for outer mainbelt asteroids. Celest. Mech. Dynam. Astron. 78, 17-46.

Lagerros, J.S.V., Müller, T.G., Klaas, U., Erikson, A., 1999. ISOPHOT polarization measurements of the asteroids (6) Hebe and (9) Metis at $2.5 \mu \mathrm{m}$. Icarus $142,454-463$.

Lemaitre, A., 1993. Proper elements: what are they? Cel. Mech. Dynam. Astron. 56, 103-119.

Levison, H.F., Duncan, M.J., 2000. Symplectically integrating close encounters with the Sun. Astron. J. 120, 2117-2123.

Martelli, G., Ryan, E.V., Nakamura, A.M., Giblin, I., 1994. Catastrophic disruption experiments: recent results. Planet. Space Sci. 42, 10131026.

Marzari, F., Davis, D., Vanzani, V., 1995. Collisional evolution of asteroid families. Icarus 113, 168-187.

Melosh, H.J., Tonks, W.B., 1993. Swapping rocks: ejection and exchange of surface material among the terrestrial planets. Meteoritics 28, 398.

Michalak, G., 2000. Determination of asteroid masses-1 Ceres, 2 Pallas, and 4 Vesta. Astron. Astrophys. 360, 363-374.

Michel, P., Benz, W., Tanga, P., Richardson, D.C., 2001. Collisions and gravitational reaccumulation: forming asteroid families and satellites. Science 294, 1696-1700

Migliorini, F., Nesvorný, D., Zappalà, V., 1998. Origins of Earth-crossing asteroids: a quantitative simulation. Science 281, 2022-2024.

Mikkola, S., 1998. Non-canonical perturbations in symplectic integrations. Celest. Mech. Dynam. Astron. 71, 243-271.

Milani, A., Knežević, Z., 1994. Asteroid proper elements and the dynamical structure of the asteroid main belt. Icarus 107, 219-254.

Morbidelli, A., Nesvorný, D., 1999. Numerous weak resonances drive asteroids toward terrestrial planets orbits. Icarus 139, 295-308.

Morbidelli, A., Zappalà, V., Moons, M., Cellino, A., Gonczi, R., 1995. Asteroid families close to mean-motion resonances: dynamical effects and physical implications. Icarus 118, 132-154.

Murray, C.D., Dermott, S., 1999. Solar System Dynamics, Cambridge, Univ. Press, Cambridge, UK

Murray, C.D., Holman, M., 1997. Diffusive chaos in the outer belt. Astron. J. 114, 1246-1259.

Murray, N., Holman, M., Potter, M., 1998. On the origin of chaos in the asteroid belt. Astron. J. 116, 2583-2589.

Nesvorný, D., Morbidelli, A., 1998. Three-body mean motion resonances and the chaotic structure of the asteroid belt. Astron. J. 116, 30293037.

Nesvorný, D., Morbidelli, A., 1999. An analytical model of three-body mean-motion resonances. Celest. Mech. Dynam. Astron. 71, 243-271.

Nesvorný, D., Morbidelli, A., Vokrouhlický, D., Bottke, W., Brož, M., 2002. The Flora family: a case of the dynamically dispersed collisional swarm? Icarus 157, 155-172.

Öpik, E.J., 1951. Collision probabilities with the planets and the distribution of interplanetary matter. Proc. R. Irish. Acad. 54A, 165-199.

Petit, J.M., Farinella, P., 1993. Modelling the outcome of high-velocity impacts between small Solar System bodies. Celest. Mech. Dynam. Astron. 57, 1-28. 
Rubincam, D.P., 1995. Asteroid orbit evolution due to thermal drag. J. Geophys. Res. 100, 1585-1594.

Scholl, H., Schmadel, L.D., Roser, S., 1987. The mass of the asteroid (10) Hygiea derived from observations of (829) Academia. Astron. Astrophys. 179, 311-316.

Spitale, J., Greenberg, R., 2001. Numerical evaluation of the general Yarkovsky effect: effects on semimajor axis. Icarus 149, 222-234.

Thomas, P.C., Belton, M.J., Carcich, S.B., Chapman, C.R., Davies, M.E., Sullivan, R., and Veverka, J., 1996. The shape of Ida. Icarus 120, 20-32. Vokrouhlický, D., 1999. A complete linear model for the Yarkovsky thermal force on spherical asteroid fragments. Astron. Astrophys. 344, 362-366.
Vokrouhlický, D., Farinella, P., 1998. The Yarkovsky seasonal effect on asteroidal fragments: a nonlinearized theory for the plane-parallel case. Astron. J. 116, 2032-2041.

Wisdom, J., Holman, M., 1991. Symplectic maps for the n-body problem. Astron. J. 102, 1528-1538.

Zappalà, V., Cellino, A., Farinella, P., Milani, A., 1994. Asteroid families 2. Extension to unnumbered multi-opposition asteroids. Astron. J. 107, $772-801$.

Zappalà, V., Bendjoya, Ph., Cellino, A., Farinella, P., Froeschié, C., 1995. Asteroid families: search of a 12,487-asteroid sample using two different clustering techniques. Icarus 116, 291-314. 